\title{
DABCO-Catalyzed Reaction of $\alpha$-Halo Carbonyl Compounds with Dimethyl Acetylenedicarboxylate: A Novel Method for the Preparation of Polysubstituted Furans and Highly \\ Functionalized $2 \boldsymbol{H}$-Pyrans \\ Mingjin Fan, ${ }^{\dagger}$ Zeyi Yan, ${ }^{\dagger}$ Weimin Liu, ${ }^{*}$ and Yongmin Liang ${ }^{*+*}$ \\ ${ }^{\dagger}$ State Key Laboratory of Applied Organic Chemistry, Lanzhou University, Lanzhou 730000, P.R. China \\ ${ }^{t}$ Lanzhou Institute of Chemical Physics, Chinese Academy of Science, Lanzhou 730000, P.R. China. \\ Liangym@1zu.edu.cn
}

\section{Supporting Information}

(I). Experimental section (pageS2).

(II). Spectroscopic Data for polySubstituted Furans and highly functionalized $2 H$-pyrans (pageS3-S56).

(III). Single-crystal X-ray crystallographic data for polySubstituted Furan 3c and highly functionalized $2 \mathrm{H}$-pyran $4 \mathrm{e}($ pageS57-S58). 


\section{(I). Experimental section.}

General Comments. Column chromatography was carried out on silica gel. Melting points were determined on a microscopic apparatus and were uncorrected. Column chromatography was carried out on silica gel. ${ }^{1} \mathrm{H}$ NMR spectra were recorded at $300 \mathrm{MHz}$ in $\mathrm{CDCl}_{3}$ and ${ }^{13} \mathrm{C} \mathrm{NMR}$ spectra was recorded at $75 \mathrm{MHz}$ in $\mathrm{CDCl}_{3}$ using TMS as internal standard. IR spectra were obtained using an FT IR spectrometer and only major peaks are reported in $\mathrm{cm}^{-1}$. Mass spectra were recorded by the EI method. All solvents used were dried without further purification.

General procedure for the reactions of dimethyl acetylenedicarboxylate with $\alpha$-halo carbonyl

compounds. DABCO $(0.1 \mathrm{mmol})$ was added to a stirred solution of $\alpha$-halo carbonyl compound (1.5mmol for $\alpha$-chloroketones, $1.0 \mathrm{mmol}$ for $\alpha$-bromoketones) in $6 \mathrm{ml}$ of $\mathrm{MeCN} / \mathrm{Et}_{2} \mathrm{O}(1: 1)$ and stirred at room temperature $\left(25-30{ }^{\circ} \mathrm{C}\right)$ for $30 \mathrm{~min}$. Anhydrous $\mathrm{K}_{2} \mathrm{CO}_{3}(1.5 \mathrm{mmol}$ for $\alpha$-chloroketones, $1.0 \mathrm{mmol}$ for $\alpha$-bromoketones)) was added, followed by dimethyl acetylenedicarboxylate $(0.5 \mathrm{mmol})$. The reaction was stirred at room temperature until thin layer chromatographic analysis showed complete consumption of the dimethyl acetylenedicarboxylate. $\mathrm{H}_{2} \mathrm{O}(10 \mathrm{ml})$ was added to the mixture and extracted thrice with $15 \mathrm{ml} \mathrm{CH}_{2} \mathrm{Cl}_{2}$. The combined extracts were washed with $\mathrm{H}_{2} \mathrm{O}$ and brine, and then dried $\left(\mathrm{MgSO}_{4}\right)$. The solvent was evaporated under reduced pressure and the residue was purified by flash chromatography on silica column to give furans 3 and $2 H$-pyrans 4 . 
(II). Spectroscopic Data for polySubstituted Furans and highly functionalized $2 \mathrm{H}$-pyrans.

3a Dimethyl 2-(thiophen-2-yl) furan-3, 4-dicarboxylate

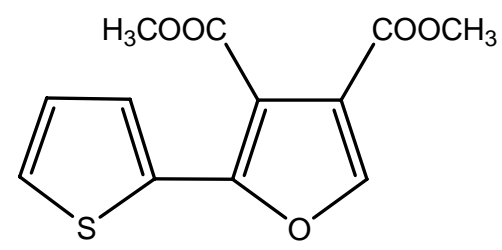

${ }^{1} \mathbf{H}$ NMR $\left(300 \mathrm{MHz} \mathrm{CDCl}_{3}\right) \delta=3.88(\mathrm{~s}, 3 \mathrm{H}), 3.95(\mathrm{~s}, 3 \mathrm{H}), 7.11(\mathrm{dd}, \mathrm{J}=4.2,4.8 \mathrm{~Hz}, 1 \mathrm{H}), 7.44(\mathrm{~d}, \mathrm{~J}=5.1 \mathrm{~Hz}$ $1 \mathrm{H}), 7.68(\mathrm{~d}, \mathrm{~J}=3.6 \mathrm{~Hz}, 1 \mathrm{H}), 7.89(\mathrm{~s}, 1 \mathrm{H})$.

${ }^{13} \mathrm{C}$ NMR $\left(75 \mathrm{MHz}, \mathrm{CDCl}_{3}\right) \delta=23.0,30.0,52.3,52.7,112.0,120.0,128.0,128.2,130.4,146.0,151.6$, 162.4, 164.0.

IR (KBr): v=3106, 2921, 1723, 1554, 1441, 1279, 1066, 707 $\mathrm{cm}^{-1}$.

MS: $\mathrm{m} / \mathrm{z}(\%)=266\left(\mathrm{M}^{+}, 50\right), 235(76.13), 111(13.45), 83(4.32)$.

HRMS calcd for $\mathrm{C}_{12} \mathrm{H}_{10} \mathrm{O}_{5} \mathrm{~S}$ : (M+Na) 289.0141, Found: 289.0147. 


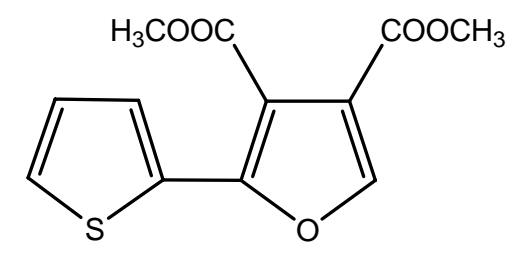



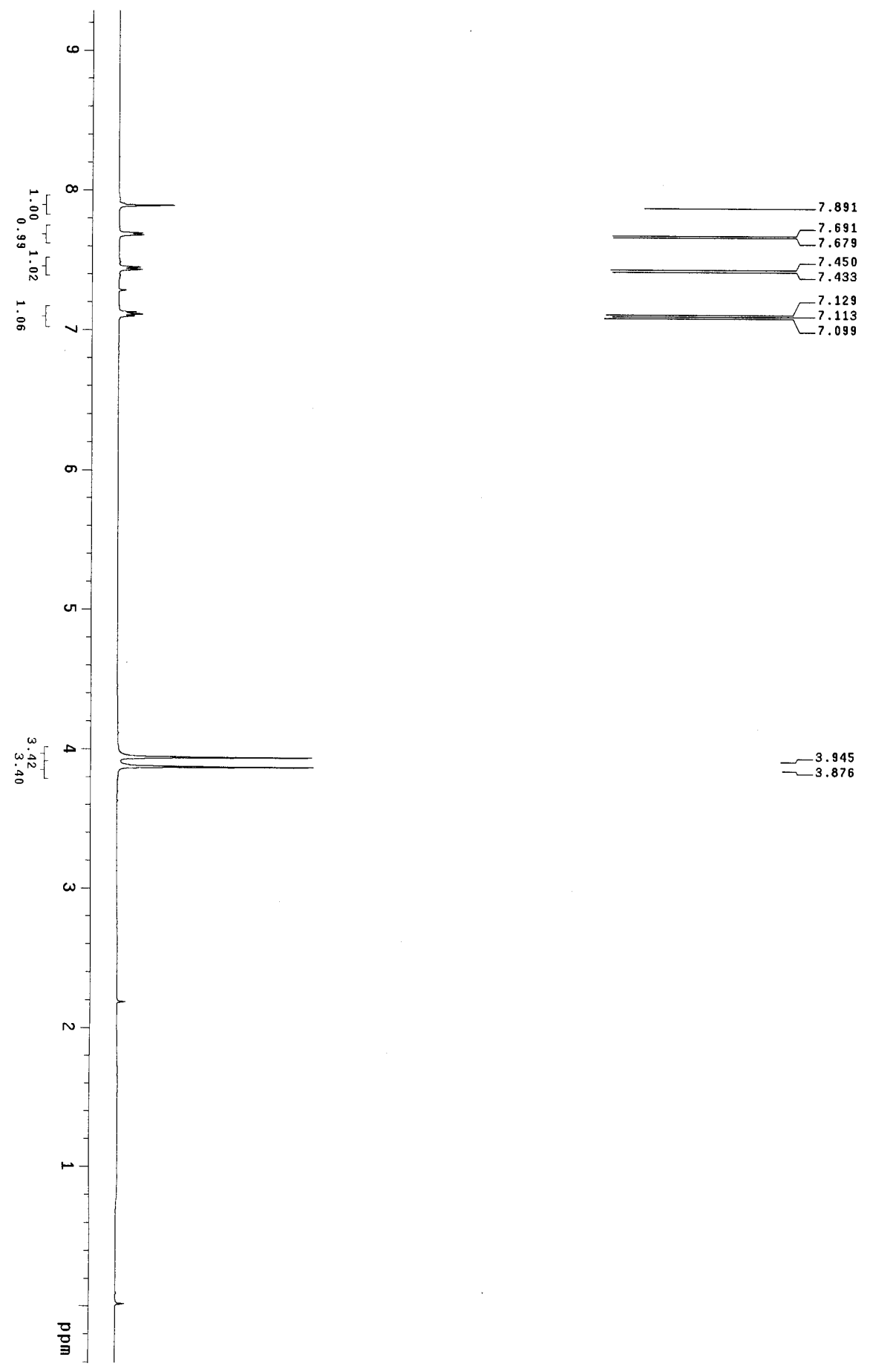

-3.945
$-\quad 3.876$ 


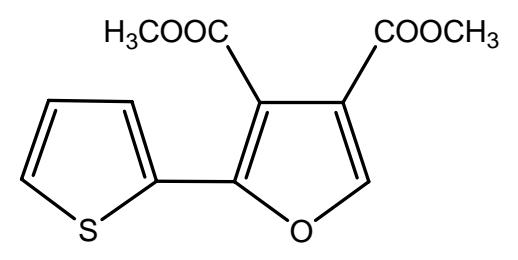



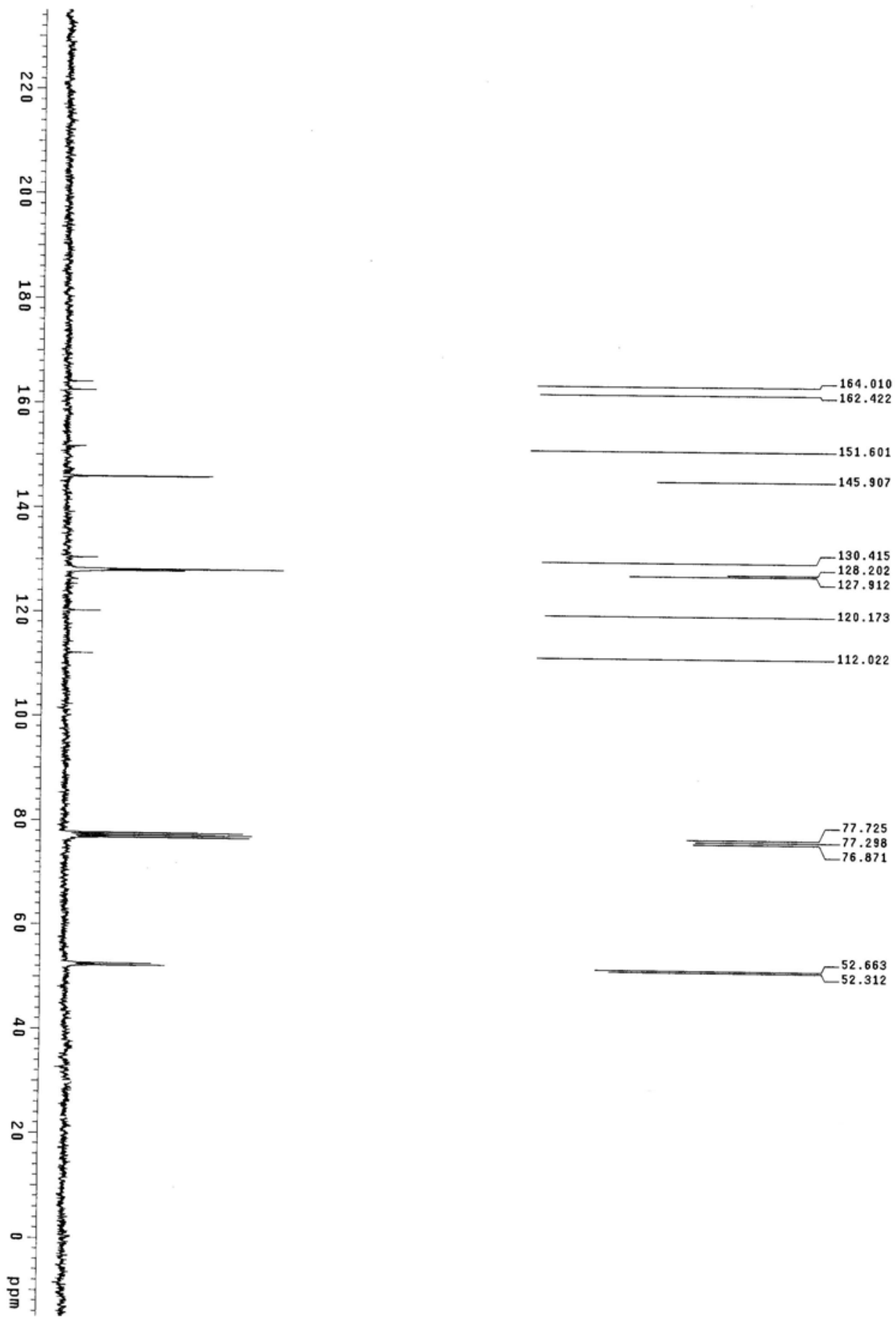

$4 a$ 


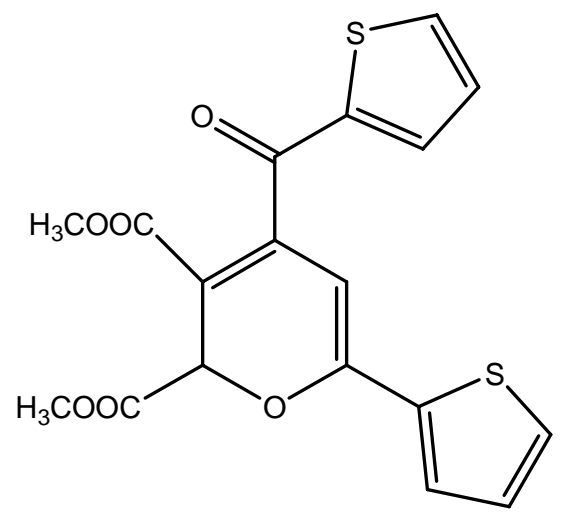

${ }^{1}$ H NMR $\left(300 \mathrm{MHz}, \mathrm{CDCl}_{3}\right) \delta=3.59(\mathrm{~s}, 3 \mathrm{H}), 3.78(\mathrm{~s}, 3 \mathrm{H}), 5.94(\mathrm{~s}, 1 \mathrm{H}), 6.01(\mathrm{~s}, 1 \mathrm{H}), 7.07-7.13(\mathrm{~m}, 2 \mathrm{H})$, $7.47(\mathrm{~d}, \mathrm{~J}=4.8 \mathrm{~Hz}, 1 \mathrm{H}), 7.53(\mathrm{~d}, \mathrm{~J}=2.7 \mathrm{~Hz}, 1 \mathrm{H}), 7.61(\mathrm{~d}, \mathrm{~J}=3.9 \mathrm{~Hz}, 1 \mathrm{H}), 7.72(\mathrm{~d}, \mathrm{~J}=4.8 \mathrm{~Hz}, 1 \mathrm{H})$.

${ }^{13} \mathrm{C}$ NMR $\left(75 \mathrm{MHz}, \mathrm{CDCl}_{3}\right) \delta=52.3,53.2,72.5,97.6,110.8,128.5,128.7,128.8,130.2,134.6,135.3$, $136.0,142.8,144.6,154.6,164.0,169.8,187.0$.

IR (KBr): v=2953, 1741, 1710, 1658, 1412, 1254, 1086, 756 $\mathrm{cm}^{-1}$.

MS: $\mathrm{m} / \mathrm{z}(\%)=390\left(\mathrm{M}^{+}, 2.92\right), 359(0.79), 331(52.84), 111(100)$.

HRMS calcd for $\mathrm{C}_{18} \mathrm{H}_{14} \mathrm{O}_{6} \mathrm{~S}_{2}$ : (M+NH4) 408.0570, Found: 408.0567. 


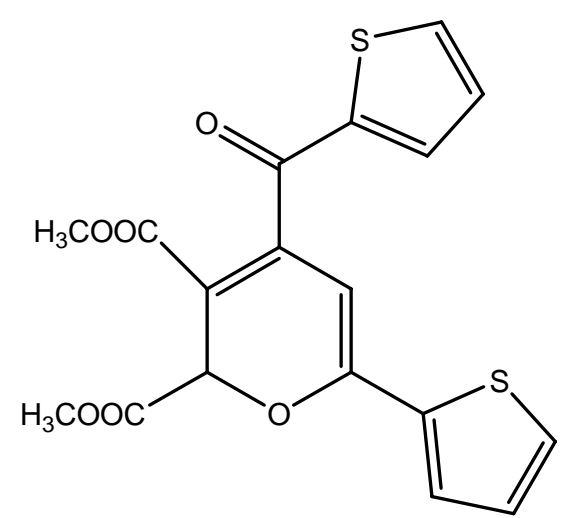



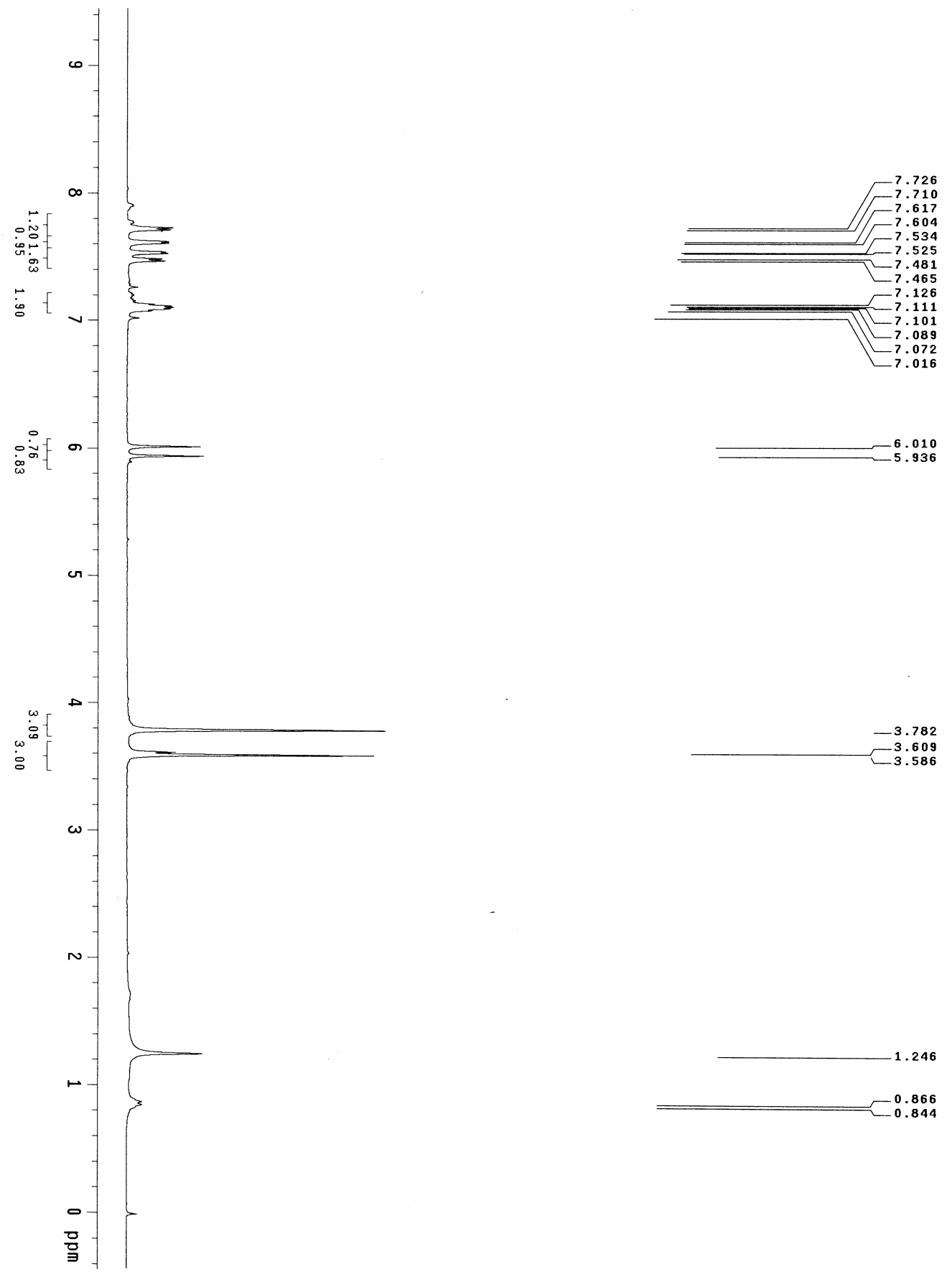


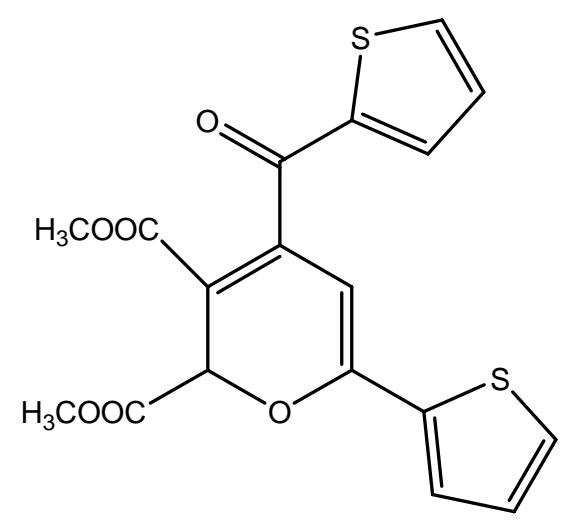



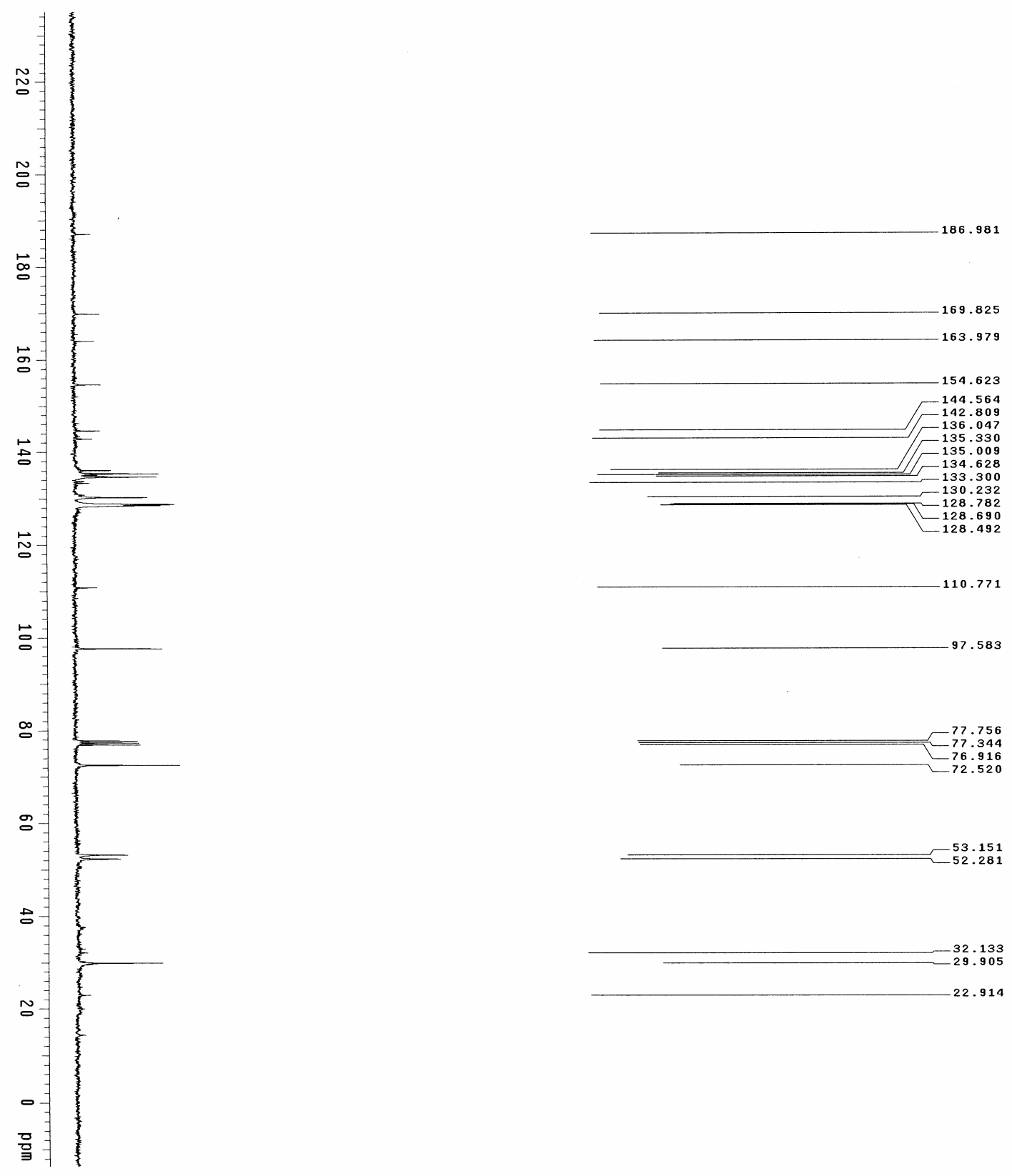

3b Dimethyl 2-(5-bromothiopen-2-yl) furan-3, 4-dicarboxylate

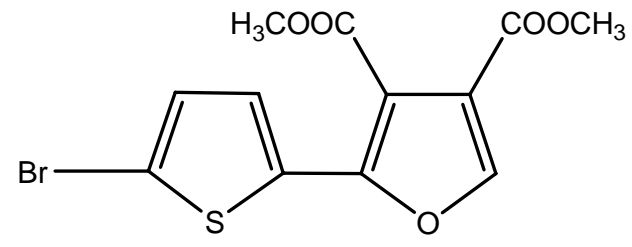


$\operatorname{mp} 68-70^{\circ} \mathrm{C}$.

${ }^{1}$ H NMR (300MHz, $\left.\mathrm{CDCl}_{3}\right) \delta=3.86(\mathrm{~s}, 3 \mathrm{H}), 3.92(\mathrm{~s}, 3 \mathrm{H}), 7.06(\mathrm{~d}, \mathrm{~J}=3.9 \mathrm{~Hz}, 1 \mathrm{H}), 7.44(\mathrm{~d}, \mathrm{~J}=3.9 \mathrm{~Hz}, 1 \mathrm{H})$, 7.86(s, 1H).

${ }^{13} \mathrm{C}$ NMR $\left(75 \mathrm{MHz}, \mathrm{CDCl}_{3}\right) \delta=52.0,52.4,111.7,116.0,120.0,128.2,130.3,131.4,145.7,150.7$, $161.9,163.4$.

MS: $\mathrm{m} / \mathrm{z}(\%)=344\left(\mathrm{M}^{+}, 5.48\right), 346(5.69), 313(7.92), 315(8.07), 265(100), 250(27.85), 191(6.63)$, 59(6.62), 45(7.92).

IR (KBr): v=2951, 1728, 1556, 1443, 1277, 1068, 812, $762 \mathrm{~cm}^{-1}$.

HRMS calcd for $\mathrm{C}_{12} \mathrm{H}_{9} \mathrm{BrO}_{5} \mathrm{~S}$ : (M+H) 344.9427, Found: 344.9434 . 


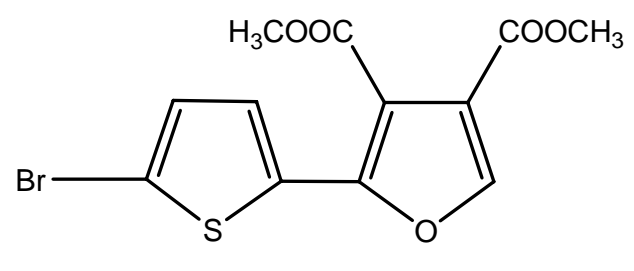



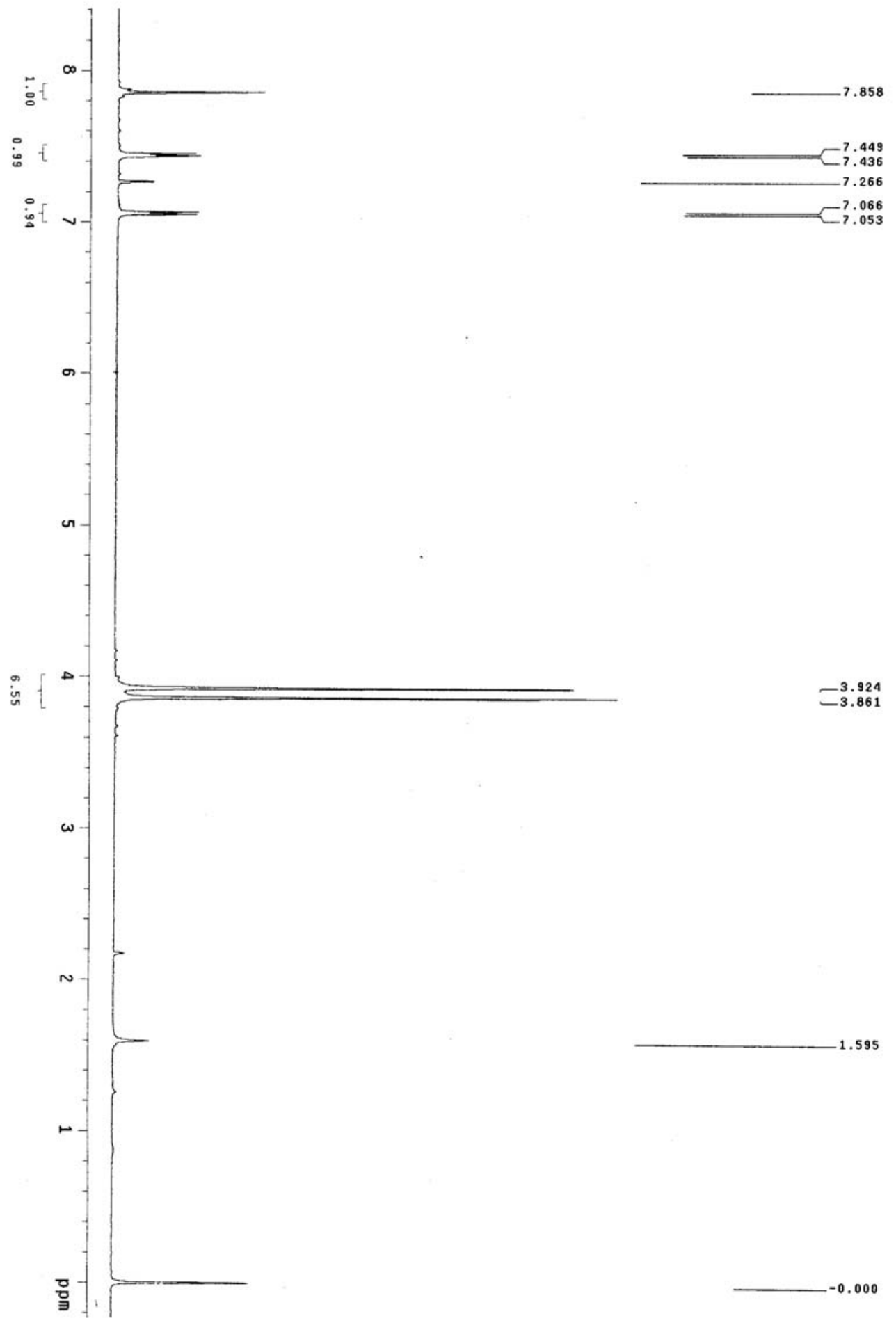

-3.924
-3.861

$-1.595$

$-0.000$ 


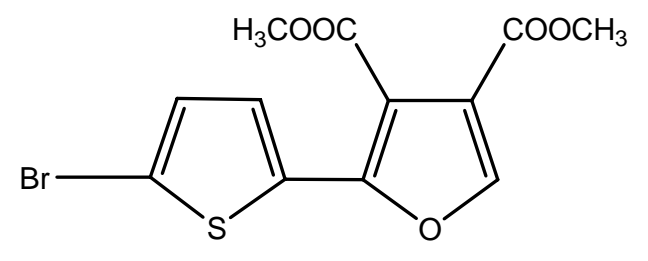



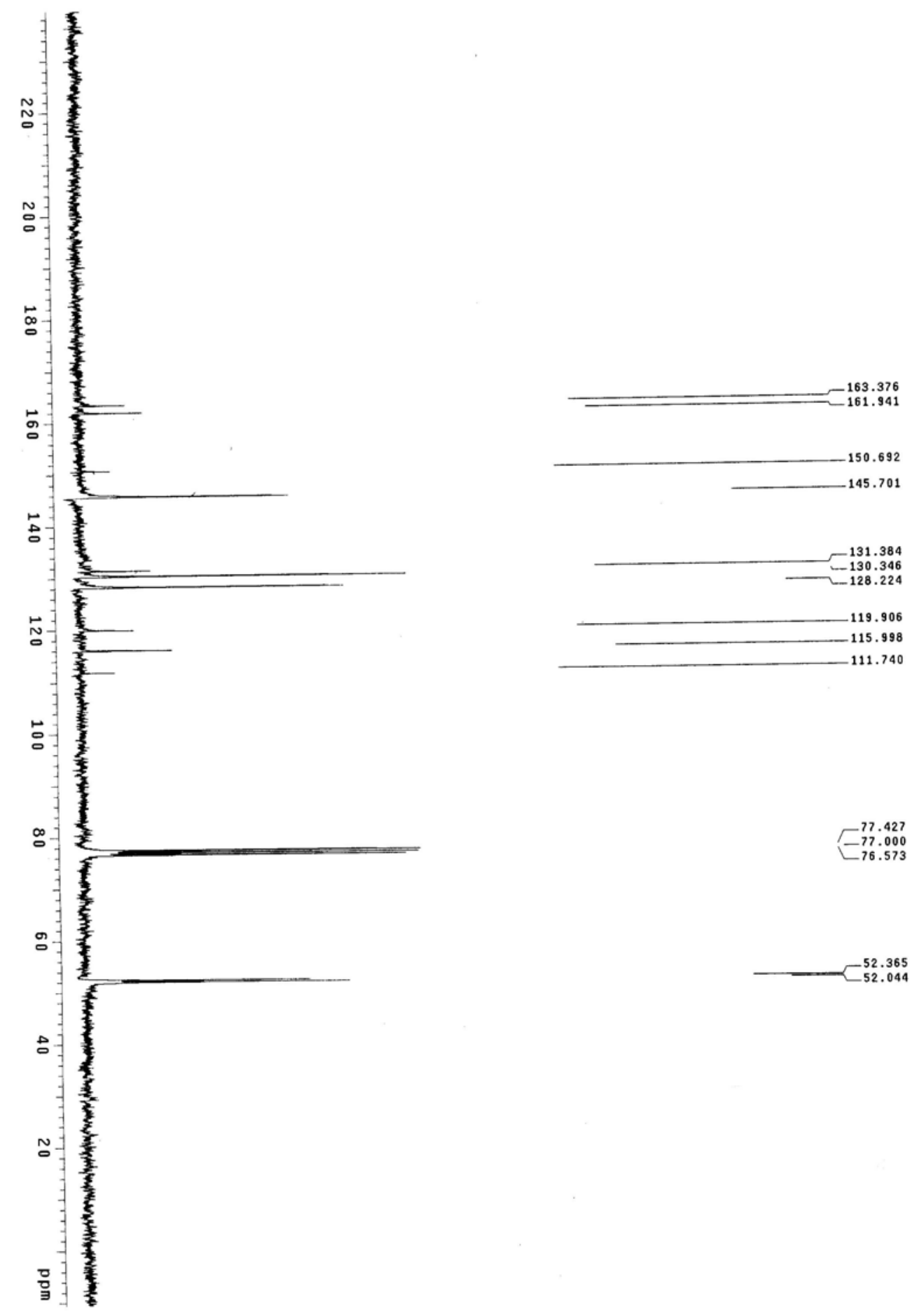


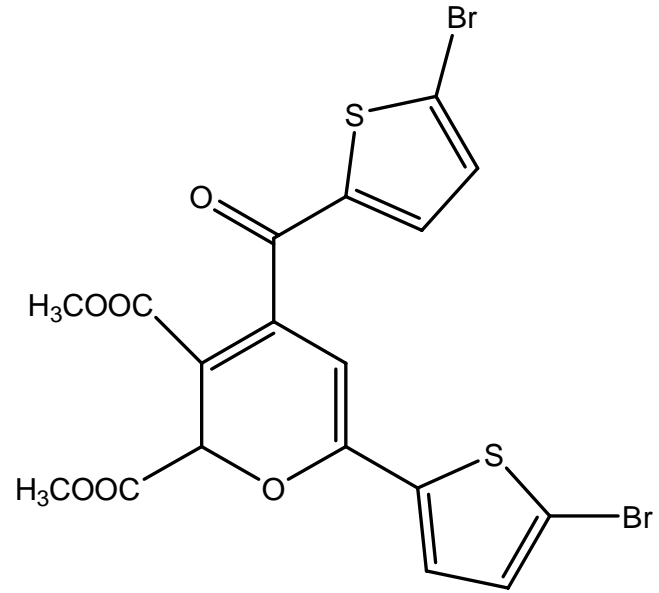

mp $136-138^{\circ} \mathrm{C}$.

${ }^{1} \mathbf{H}$ NMR $\left(300 \mathrm{MHz}, \mathrm{CDCl}_{3}\right) \delta=3.64(\mathrm{~s}, 3 \mathrm{H}), 3.79(\mathrm{~s}, 3 \mathrm{H}), 5.83(\mathrm{~s}, 1 \mathrm{H}), 5.96(\mathrm{~s}, 1 \mathrm{H}), 7.05-7.35(\mathrm{~m}, 4 \mathrm{H})$.

${ }^{13} \mathbf{C}$ NMR $\left(75 \mathrm{MHz}, \mathrm{CDCl}_{3}\right) \delta=52.5,53.3,72.5,97.5,111.6,118.2,124.5,128.7,131.5,131.9,134.7$, $137.2,143.5,144.0,153.5,163.7,169.6,185.6$.

MS: $\mathrm{m} / \mathrm{z}(\%)=546\left(\mathrm{M}^{+}, 2.57\right), 548(5.88), 550(3.84), 487(47.56), 489(100), 491(56.96) 189(87.74)$, 191(84.84).

IR $(\mathrm{KBr}): \mathrm{v}=2952,1746,1710,1655,1409,1243,1079,731 \mathrm{~cm}^{-1}$.

HRMS calcd for $\mathrm{C}_{18} \mathrm{H}_{12} \mathrm{Br}_{2} \mathrm{O}_{6} \mathrm{~S}_{2}:(\mathrm{M}+\mathrm{H})$ 546.8515, Found: 546.8524 . 


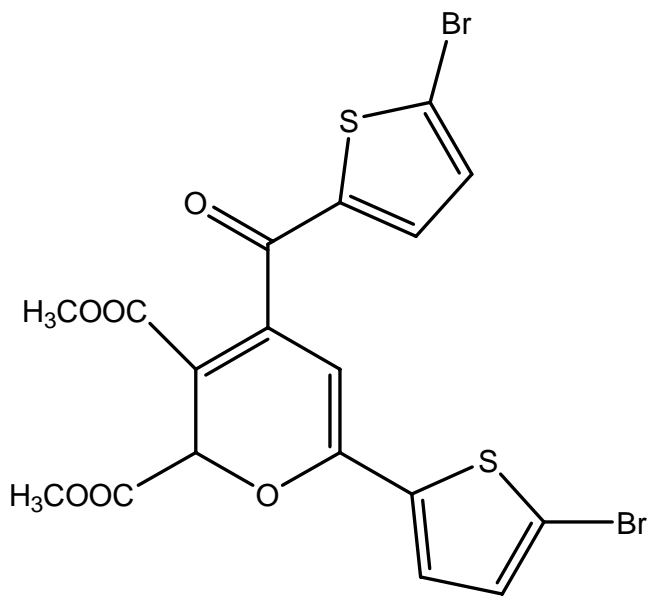



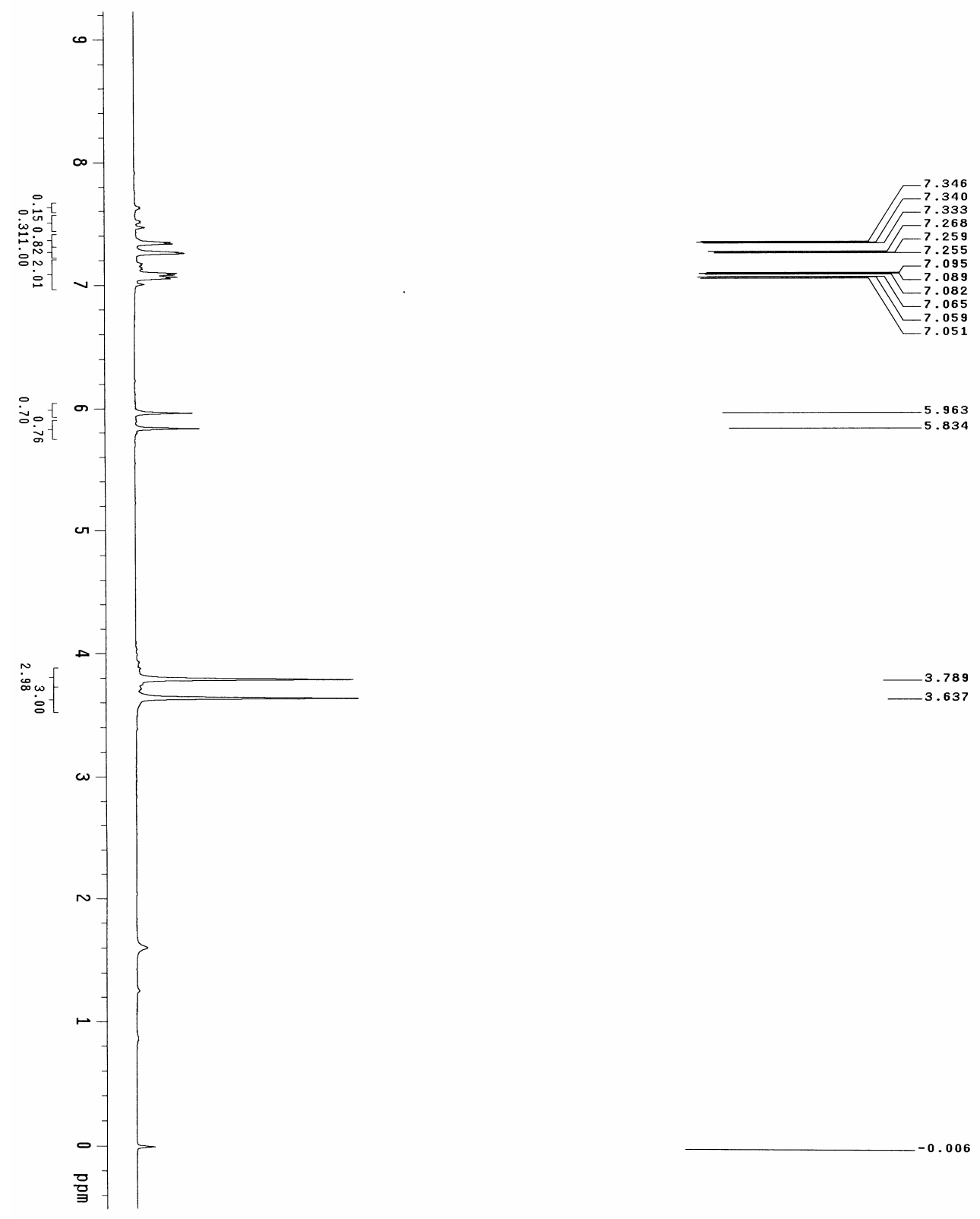


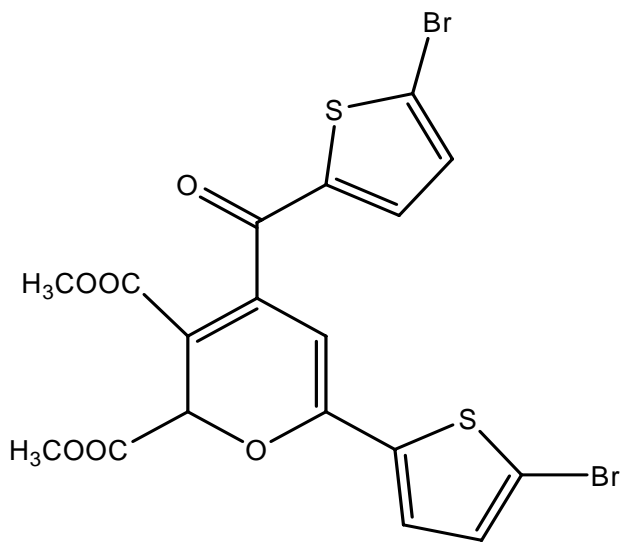



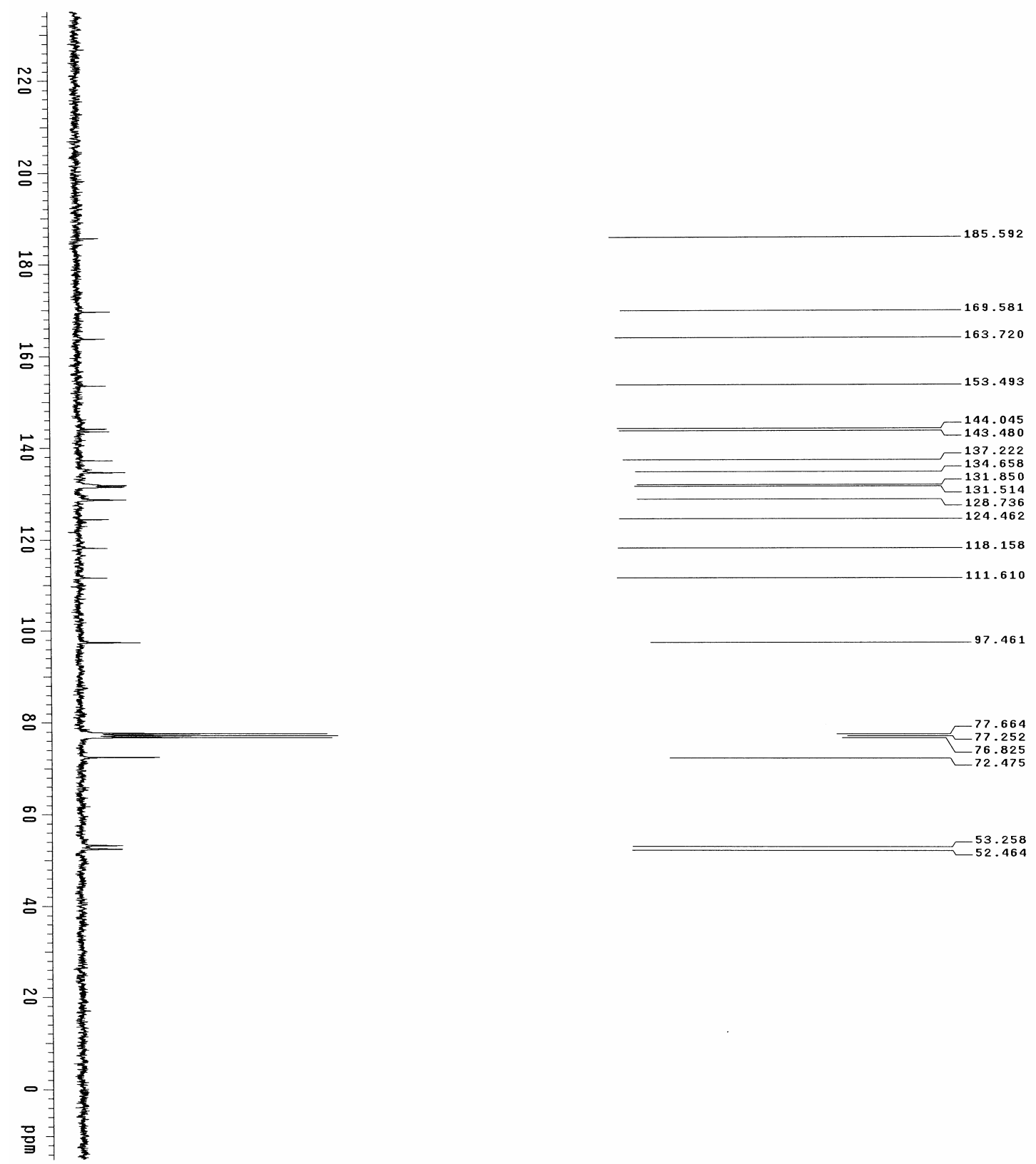

3c Dimethyl 2-phenylfuran-3, 4-dicarboxylate

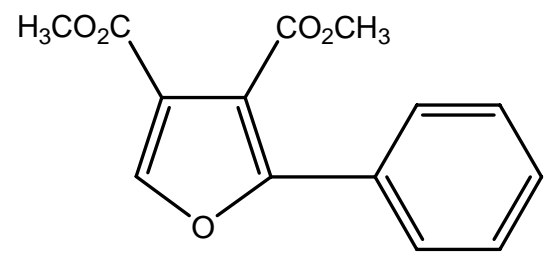


$\operatorname{mp} 68-70^{\circ} \mathrm{C}$.

${ }^{1} \mathbf{H}$ NMR $\left(300 \mathrm{MHz}, \mathrm{CDCl}_{3}\right) \delta=3.87(\mathrm{~d}, \mathrm{~J}=3.6 \mathrm{~Hz}, 3 \mathrm{H}), 3.92(\mathrm{~d}, \mathrm{~J}=3.6 \mathrm{~Hz}, 3 \mathrm{H}), 7.40-7.43(\mathrm{~m}, 3 \mathrm{H})$, 7.69-7.73(m, 2H), 7.98(d, J=3.0Hz, 1H).

${ }^{13} \mathrm{C}$ NMR $\left(75 \mathrm{MHz}, \mathrm{CDCl}_{3}\right) \delta=52.2,53.0,113.8,120.0,126.6,129.0,129.7,146.5,154.3,162.5$, 165.1.

IR (KBr): v=3151, 2955, 1717, 1552, 1441, 1282, 1151, 771, $693 \mathrm{~cm}^{-1}$.

MS: $\mathrm{m} / \mathrm{z}(\%)=260\left(\mathrm{M}^{+}, 70.80\right), 229(100), 105(16.89), 77(26.31)$.

HRMS calcd for $\mathrm{C}_{14} \mathrm{H}_{12} \mathrm{O}_{5}$ : (M+Na) 283.0577, Found: 283.0583. 


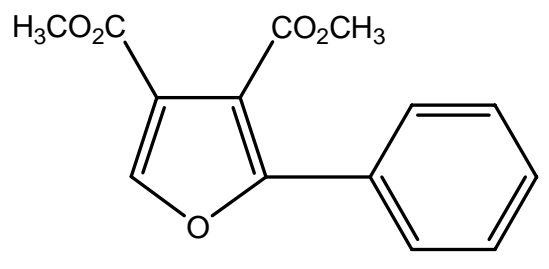



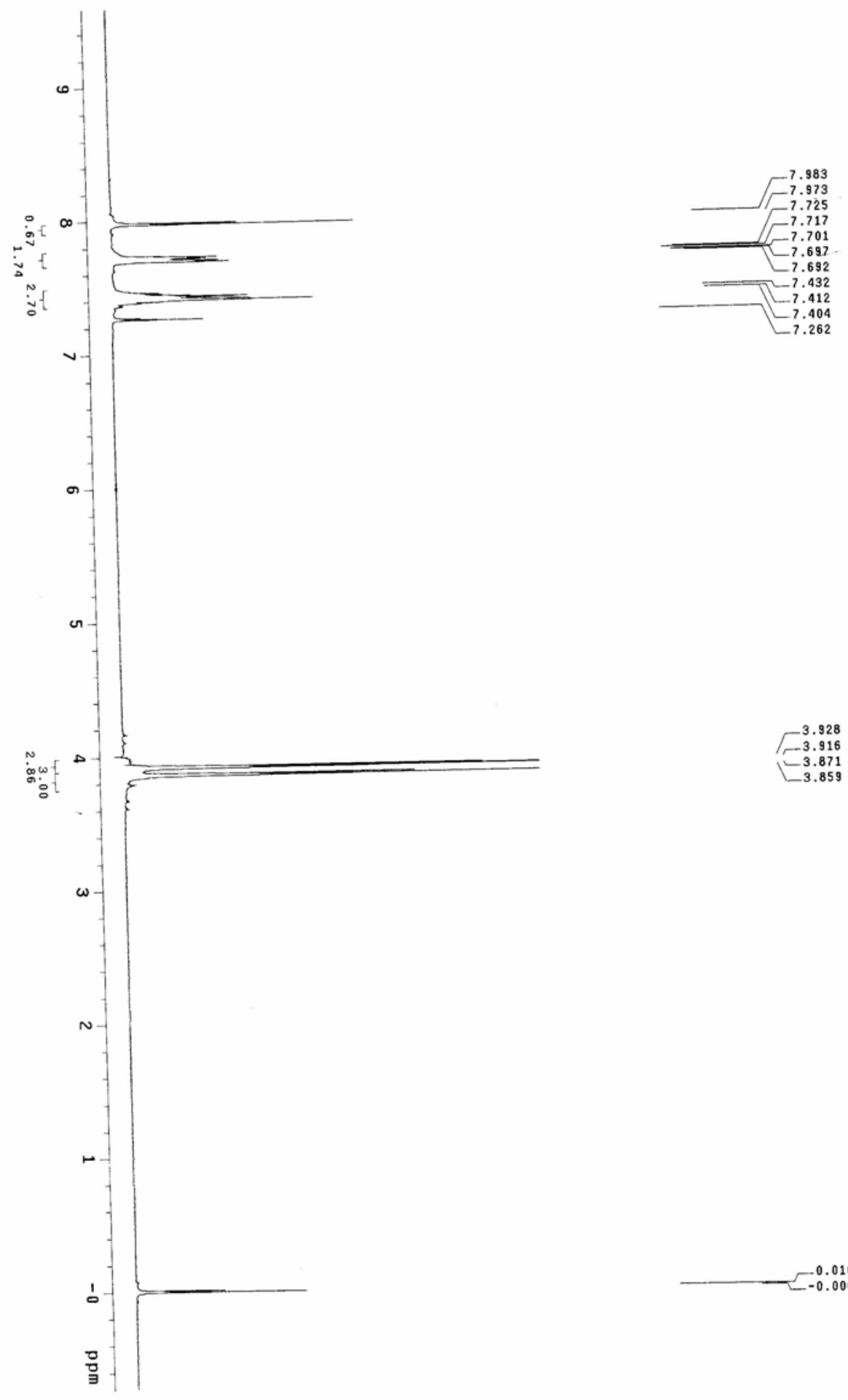

-3.871
-3.859

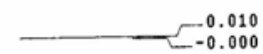




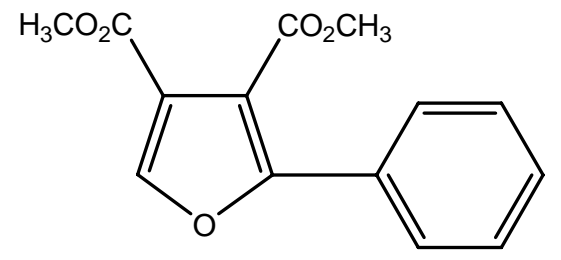



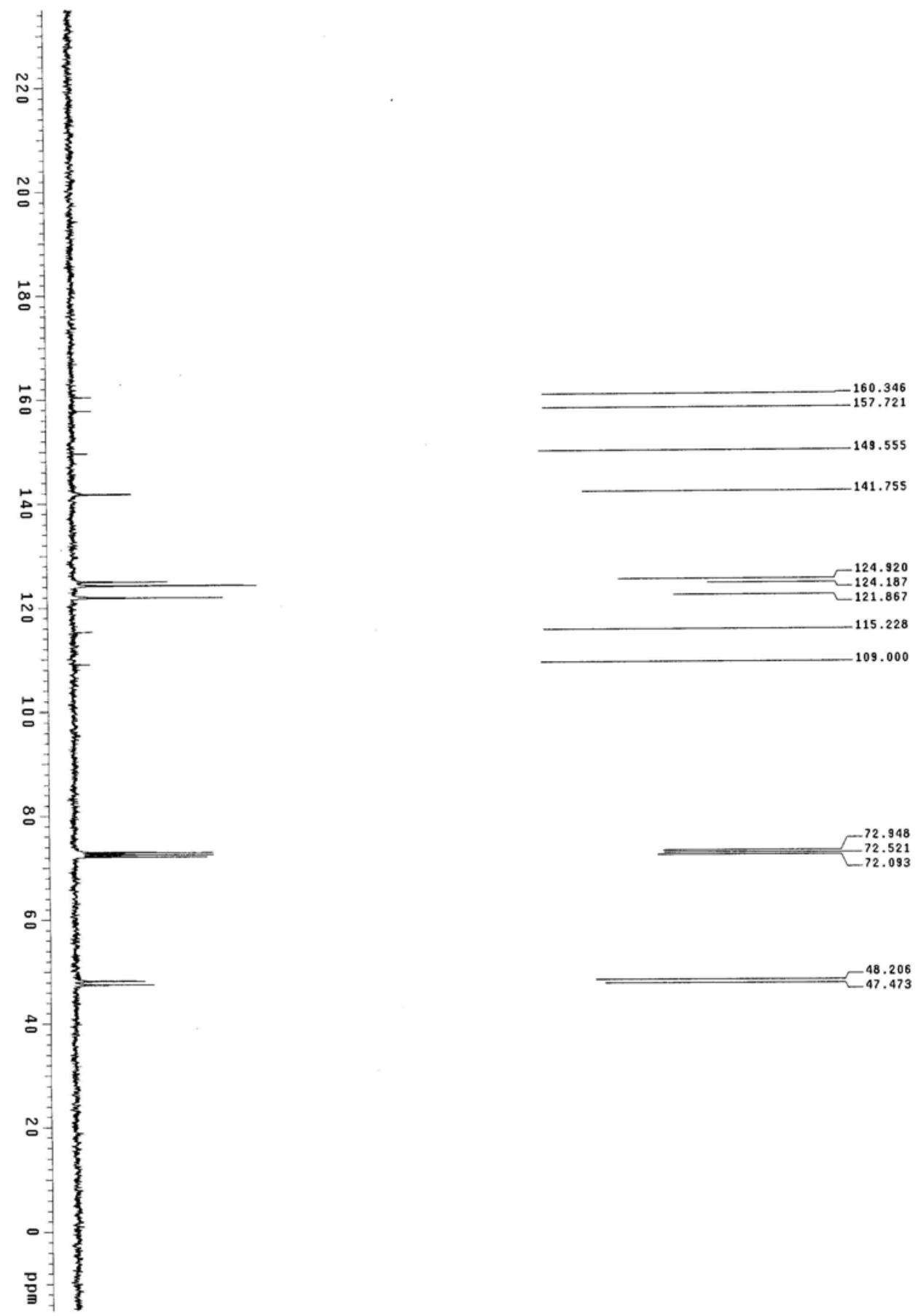


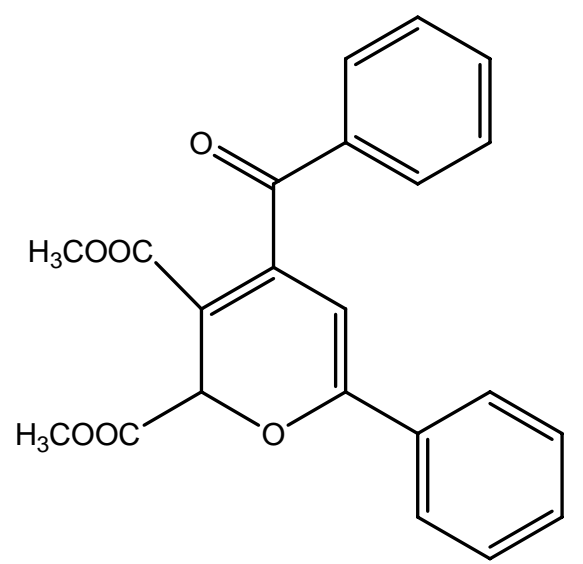

mp $99-101^{\circ} \mathrm{C}$.

${ }^{1}$ H NMR $\left(300 \mathrm{MHz}, \mathrm{CDCl}_{3}\right) \delta=3.52(\mathrm{~s}, 3 \mathrm{H}), 3.78(\mathrm{~s}, 3 \mathrm{H}), 6.01(\mathrm{~s}, 1 \mathrm{H}), 6.09(\mathrm{~s}, 1 \mathrm{H}), 7.37-7.95(\mathrm{~m}, 10 \mathrm{H})$.

${ }^{13} \mathrm{C}$ NMR $\left(75 \mathrm{MHz}, \mathrm{CDCl}_{3}\right) \delta=51.7,52.7,72.0,97.9,110.6,126.8,128.4,128.7,130.9,131.7,133.7$, 135.0, 144.9, 159.1, 163.7, 169.6, 194.6.

IR $(\mathrm{KBr}): \mathrm{v}=2954,1747,1708,1679,1254,1084,730,695 \mathrm{~cm}^{-1}$ MS: $\mathrm{m} / \mathrm{z}(\%)=378\left(\mathrm{M}^{+}, 1.11\right), 319(43.14), 105(81.62), 77(100)$.

HRMS calcd for $\mathrm{C}_{22} \mathrm{H}_{18} \mathrm{O}_{6}$ : (M+Na) 401.0996, Found: 401.0990. 

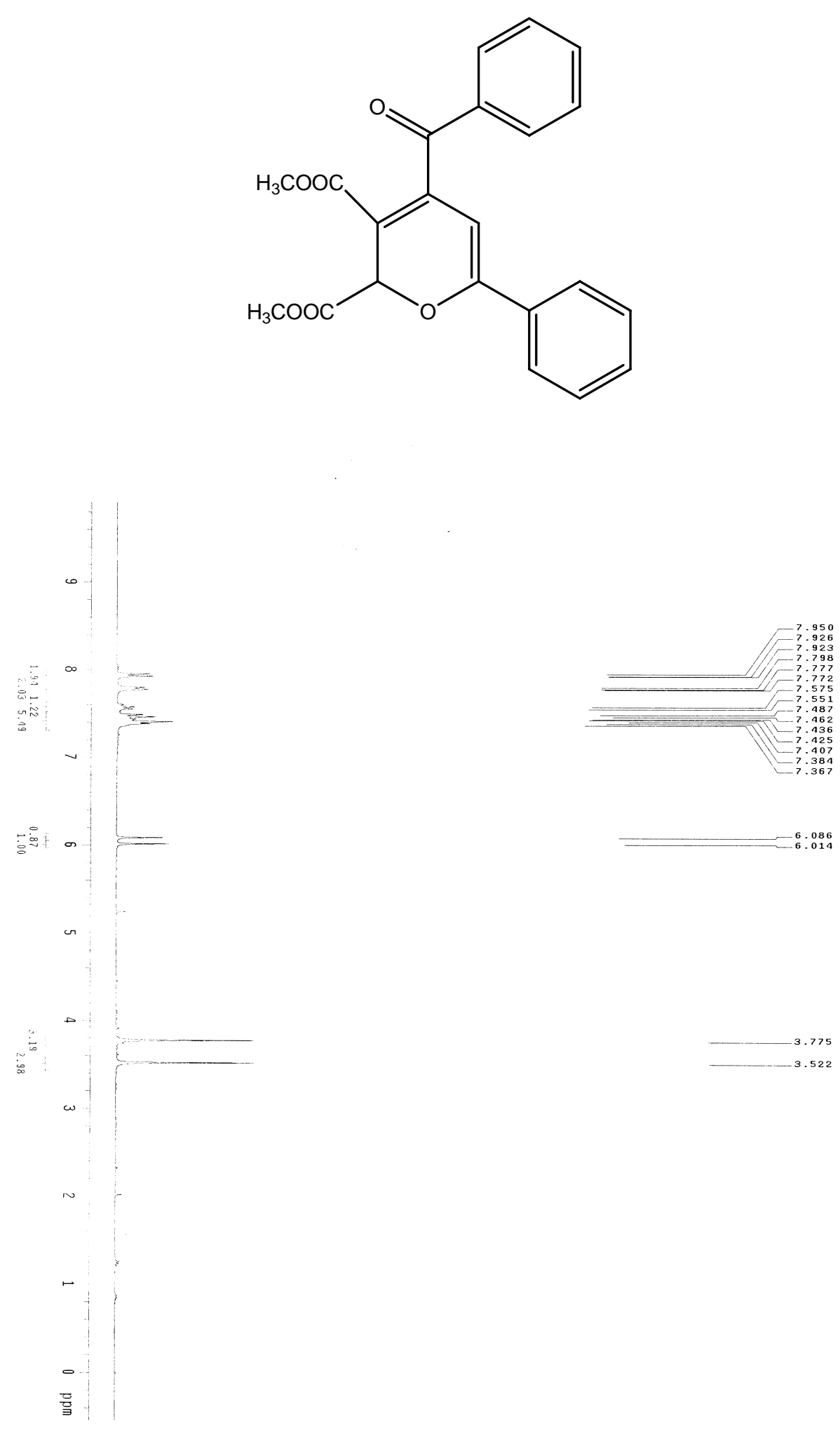


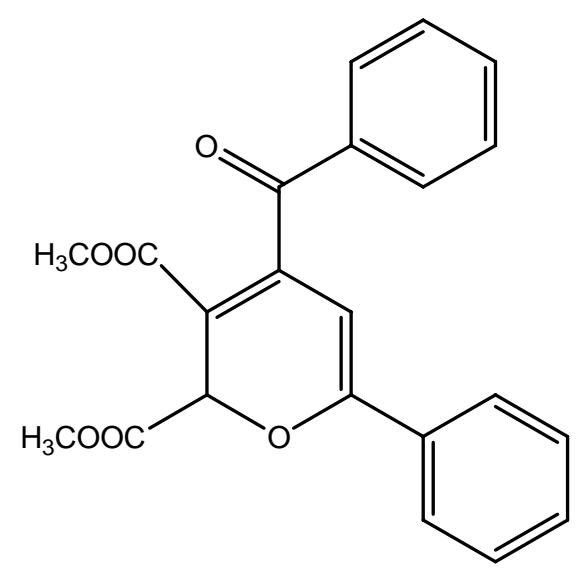



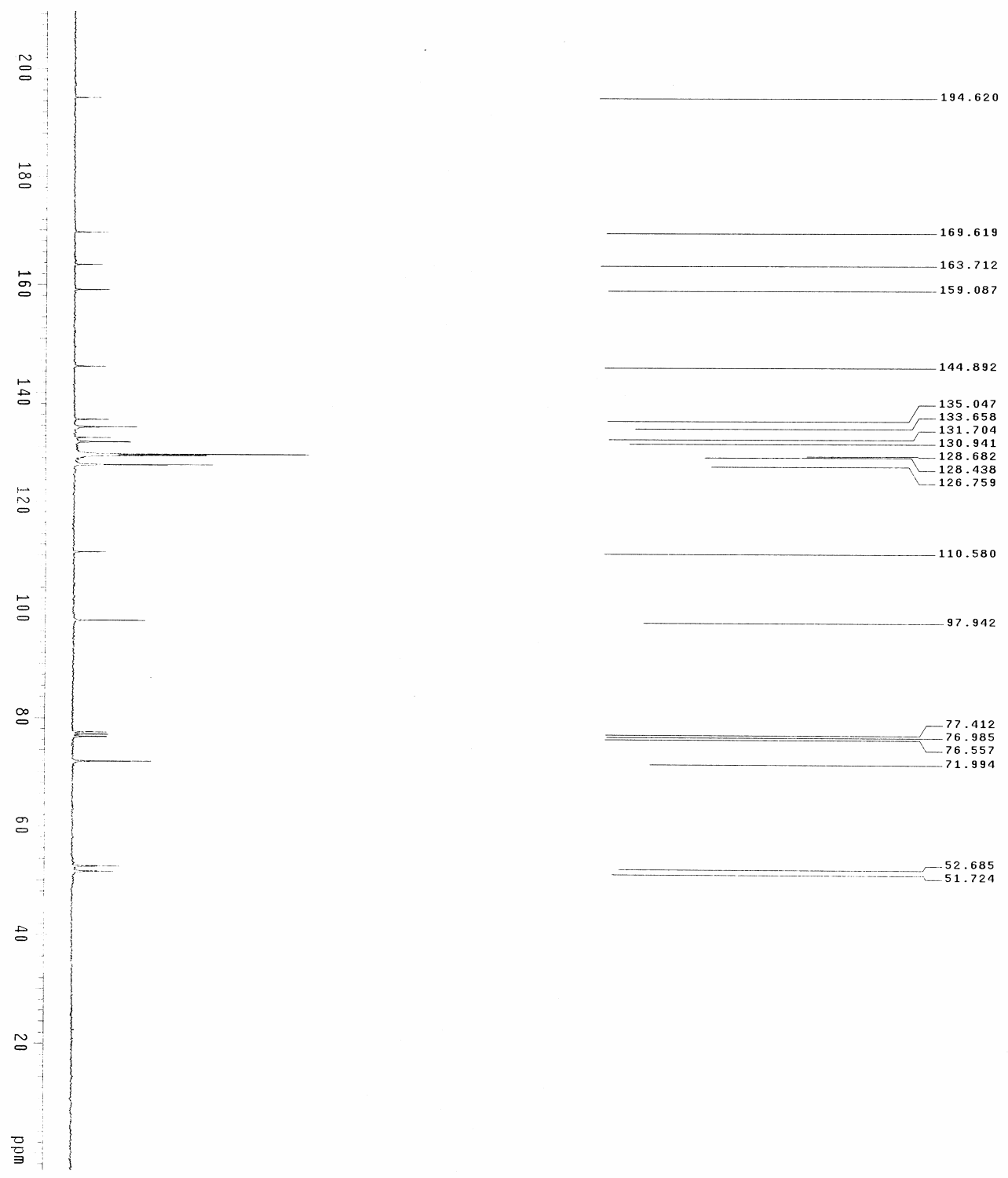

3d Dimethyl 2-(4-bromophenyl) furan-3, 4-dicarboxylate

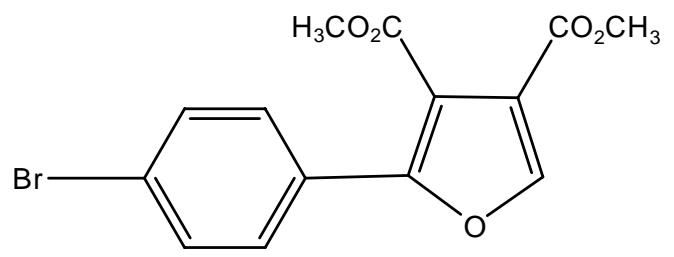

mp $79-81^{\circ} \mathrm{C}$. 
${ }^{1}$ H NMR $\left(300 \mathrm{MHz}, \mathrm{CDCl}_{3}\right) \delta=3.85(\mathrm{~s}, 3 \mathrm{H}), 3.91(\mathrm{~s}, 3 \mathrm{H}), 7.56(\mathrm{~m}, 4 \mathrm{H}), 7.96(\mathrm{~s}, 1 \mathrm{H})$.

${ }^{13}$ C NMR $\left(75 \mathrm{MHz}, \mathrm{CDCl}_{3}\right) \delta=52.3,53.0,114.2,120.2,124.1,127.8,128.2,132.2,146.7,153.4$, $162.3,164.8$

IR (KBr): v=3163, 2951, 1733, 1547, 1487, 1325, 1167, 1059, $816 \mathrm{~cm}^{-1}$.

MS: $\mathrm{m} / \mathrm{z}(\%)=338\left(\mathrm{M}^{+}, 100\right), 340(93.87), 307(85.46), 309(80.56), 183(19.42), 185(19.98) 155(13.95)$, 157(13.72), 113(43.45), 59(25.64).

HRMS calcd for $\mathrm{C}_{14} \mathrm{H}_{11} \mathrm{BrO}_{5}$ : (M+Na) 360.9682, Found: 360.9677 .

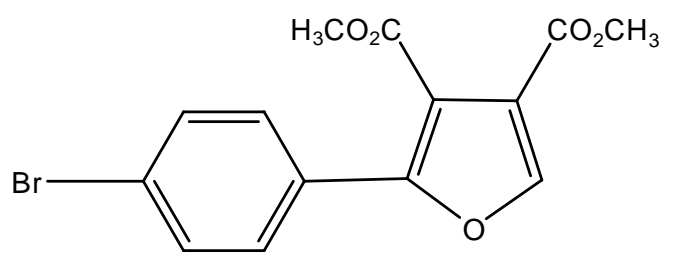



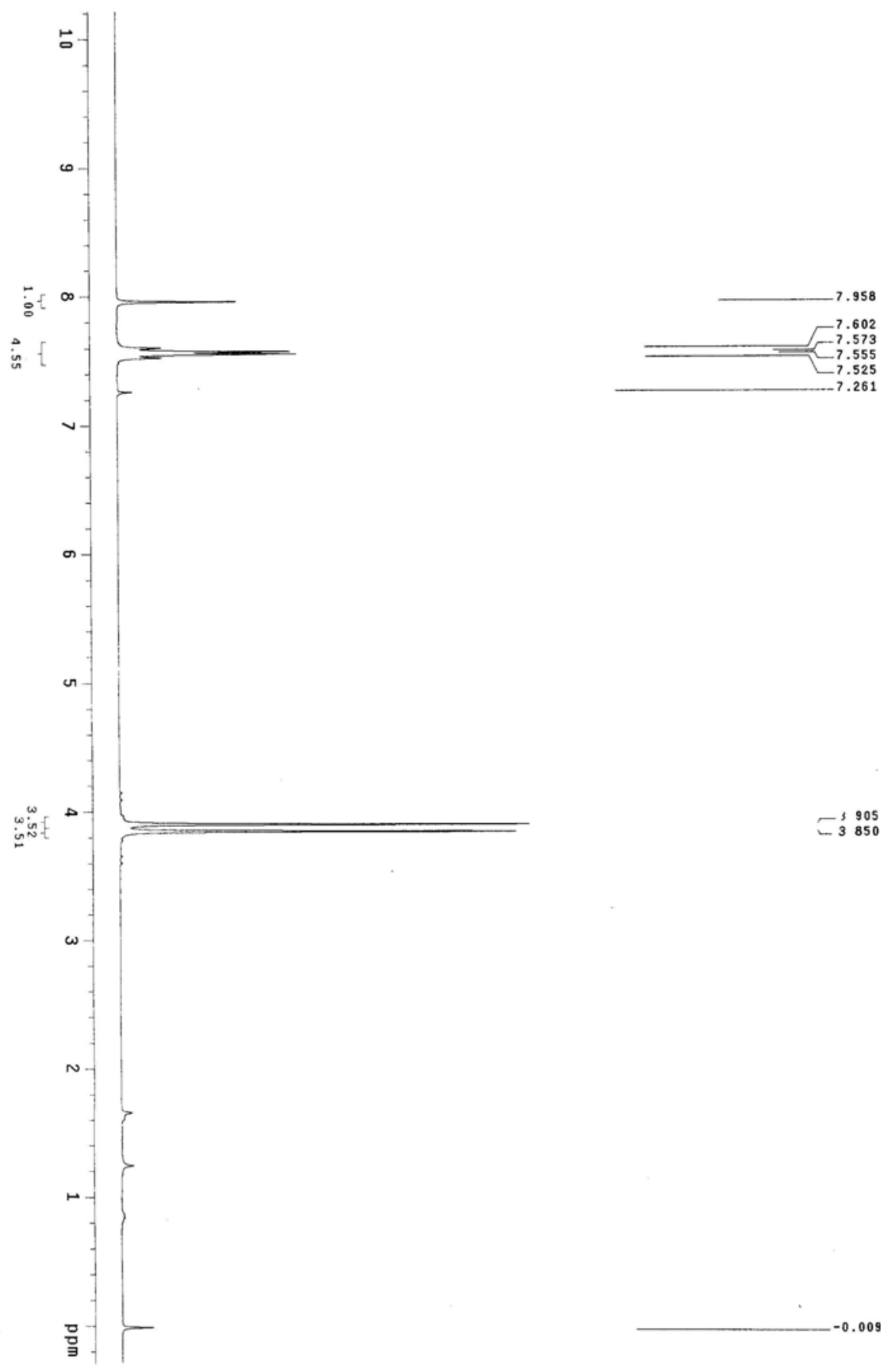

-3905
-3850

$-0.009$ 


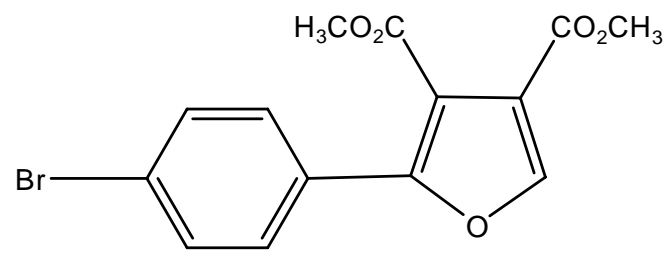




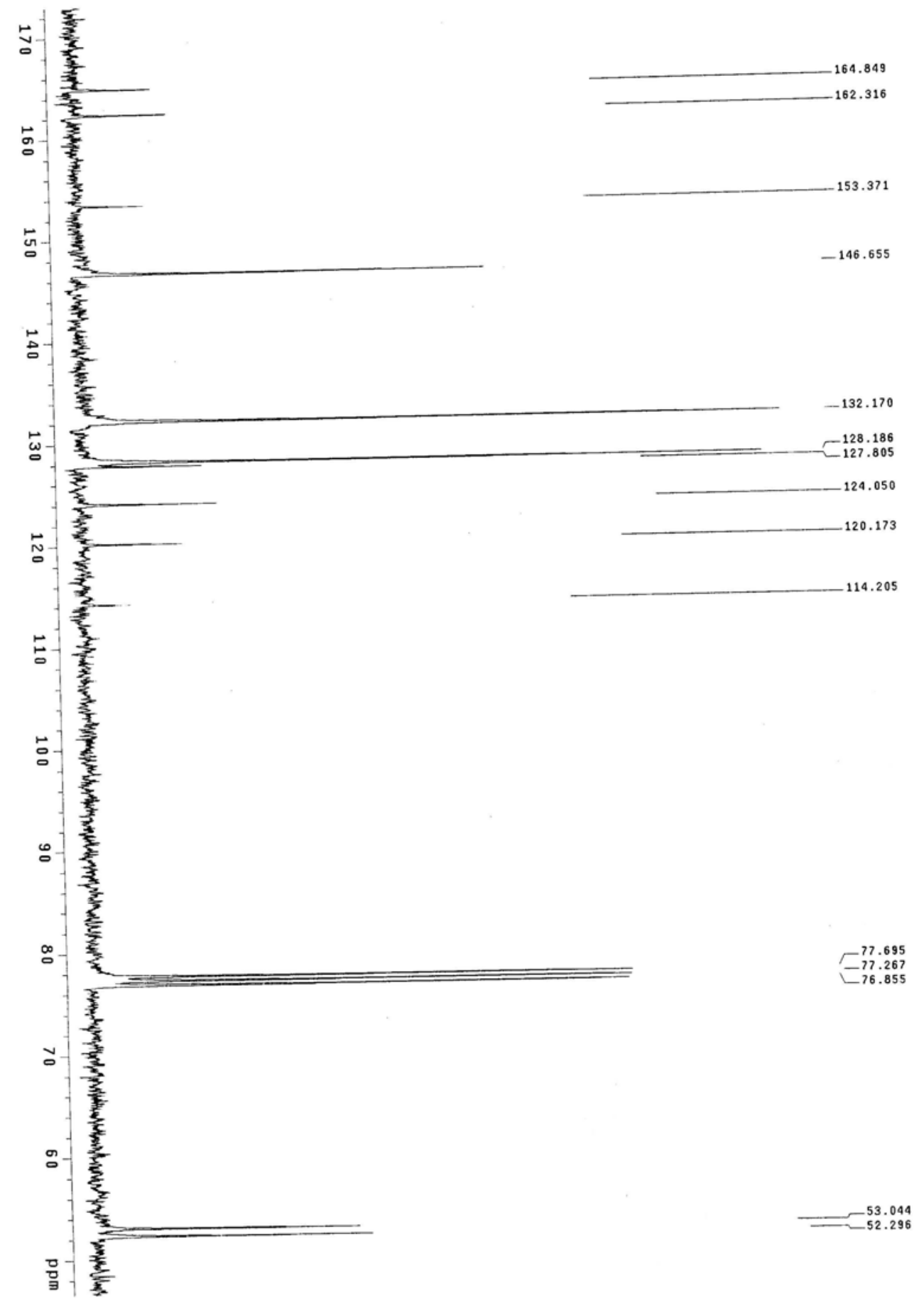




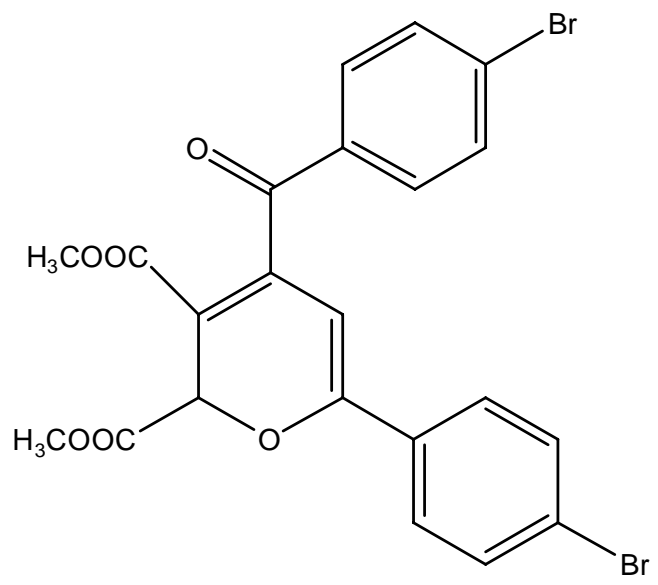

mp $142-143^{\circ} \mathrm{C}$

${ }^{1}$ H NMR $\left(300 \mathrm{MHz}, \mathrm{CDCl}_{3}\right) \delta=3.57(\mathrm{~s}, 3 \mathrm{H}), 3.79(\mathrm{~s}, 3 \mathrm{H}), 5.96(\mathrm{~s}, 1 \mathrm{H}), 6.04(\mathrm{~s}, 1 \mathrm{H}), 7.53-7.80(\mathrm{~m}, 8 \mathrm{H})$.

${ }^{13} \mathbf{C}$ NMR $\left(75 \mathrm{MHz}, \mathrm{CDCl}_{3}\right) \delta=52.1,53.0,72.1,98.2,111.4,125.8,128.3,129.2,130.3,130.7,131.9$, $132.2,134.0,144.3,158.4,163.7,169.6,193.6$.

IR $(\mathrm{KBr}): \mathrm{v}=2954,1747,1709,1680,1254,1076,839 \mathrm{~cm}^{-1}$.

MS: $\mathrm{m} / \mathrm{z}(\%)=534\left(\mathrm{M}^{+}, 0.37\right), 536(0.76), 475(15.32), 477(29.26), 479(14.98), 183(100), 185(97.86)$ 155(84.90), 157(78.49).

HRMS calcd for $\mathrm{C}_{22} \mathrm{H}_{16} \mathrm{Br}_{2} \mathrm{O}_{6}$ : $(\mathrm{M}+\mathrm{H})$ 534.9386, Found: 534.9376 . 


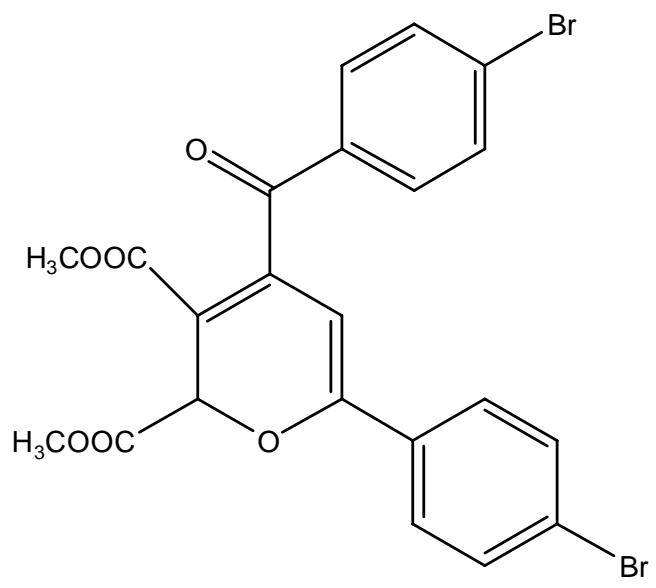



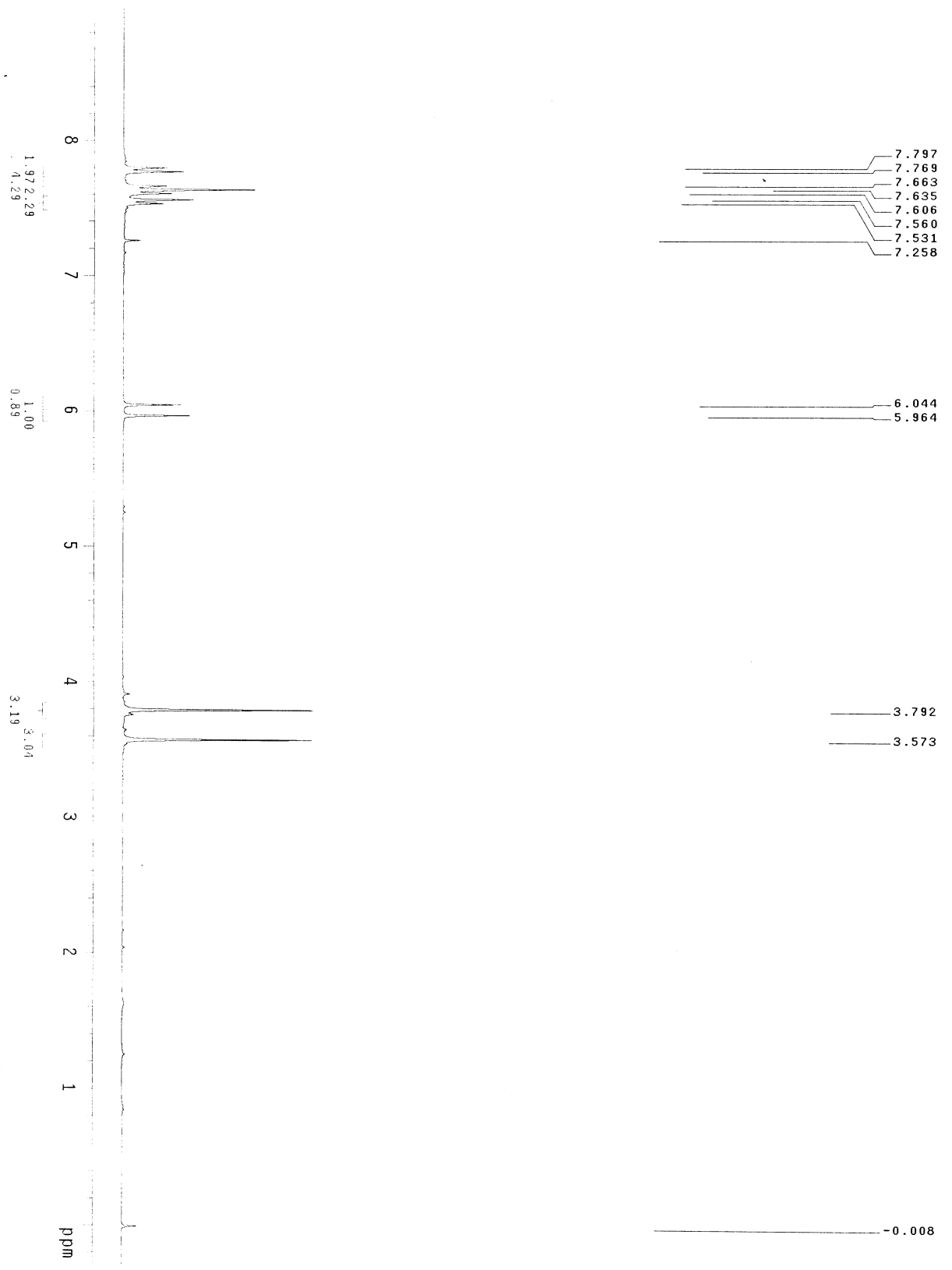


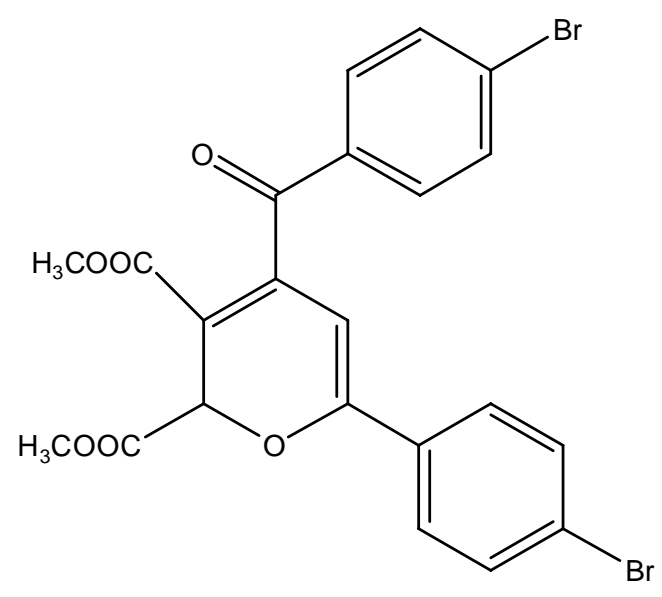



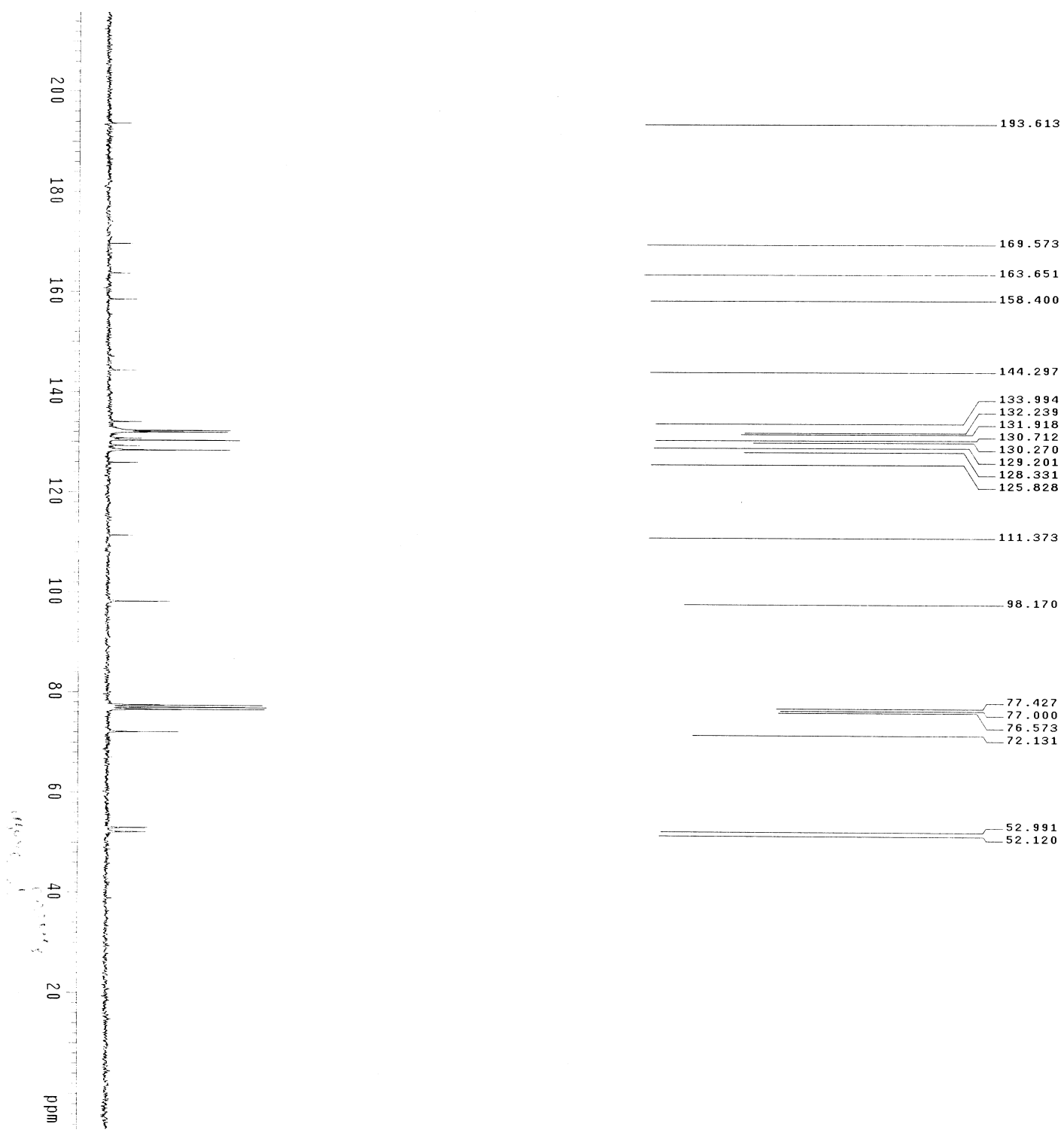

3e Dimethyl 2-(4-methylphenyl) furan-3, 4-dicarboxylate

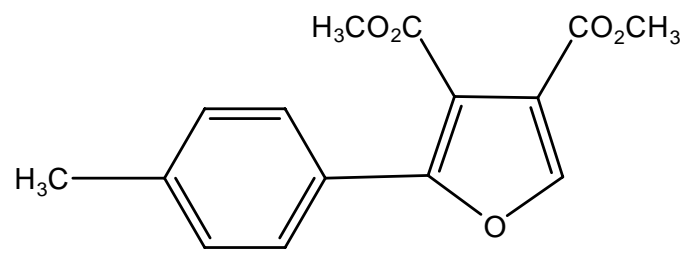

mp $95-97^{\circ} \mathrm{C}$. 
${ }^{1} \mathbf{H}$ NMR $\left(300 \mathrm{MHz}, \mathrm{CDCl}_{3}\right) \delta=2.37(\mathrm{~s}, 3 \mathrm{H}), 3.84(\mathrm{~s}, 3 \mathrm{H}), 3.90(\mathrm{~s}, 3 \mathrm{H}), 7.22(\mathrm{~d}, \mathrm{~J}=8.1 \mathrm{~Hz}, 2 \mathrm{H}), 7.59(\mathrm{~d}$, $\mathrm{J}=7.8 \mathrm{~Hz}, 2 \mathrm{H}), 7.93(\mathrm{~s}, 1 \mathrm{H})$.

${ }^{13} \mathrm{C}$ NMR $\left(300 \mathrm{MHz}, \mathrm{CDCl}_{3}\right) \delta=21.6,52.2,52.9,113.1,119.9,126.2,126.6,129.6,139.9,146.2,154.7$, $162.5,165.2$.

IR $(\mathrm{KBr}): \mathrm{v}=3158,2954,1723,1508,1441,1328,1162,1060,821 \mathrm{~cm}^{-1}$.

MS: $\mathrm{m} / \mathrm{z}(\%)=274\left(\mathrm{M}^{+}, 100\right), 243(94.1), 215(6.12), 187(6.04), 119(20.63), 91$ (24.47), 43(43.33).

HRMS calcd for $\mathrm{C}_{15} \mathrm{H}_{14} \mathrm{O}_{5}$ : $(\mathrm{M}+\mathrm{Na})$ 297.0733, Found: 297.0735.

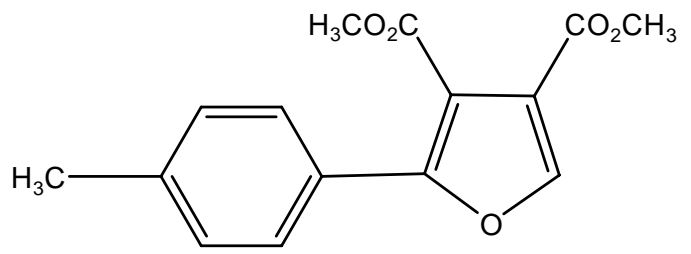



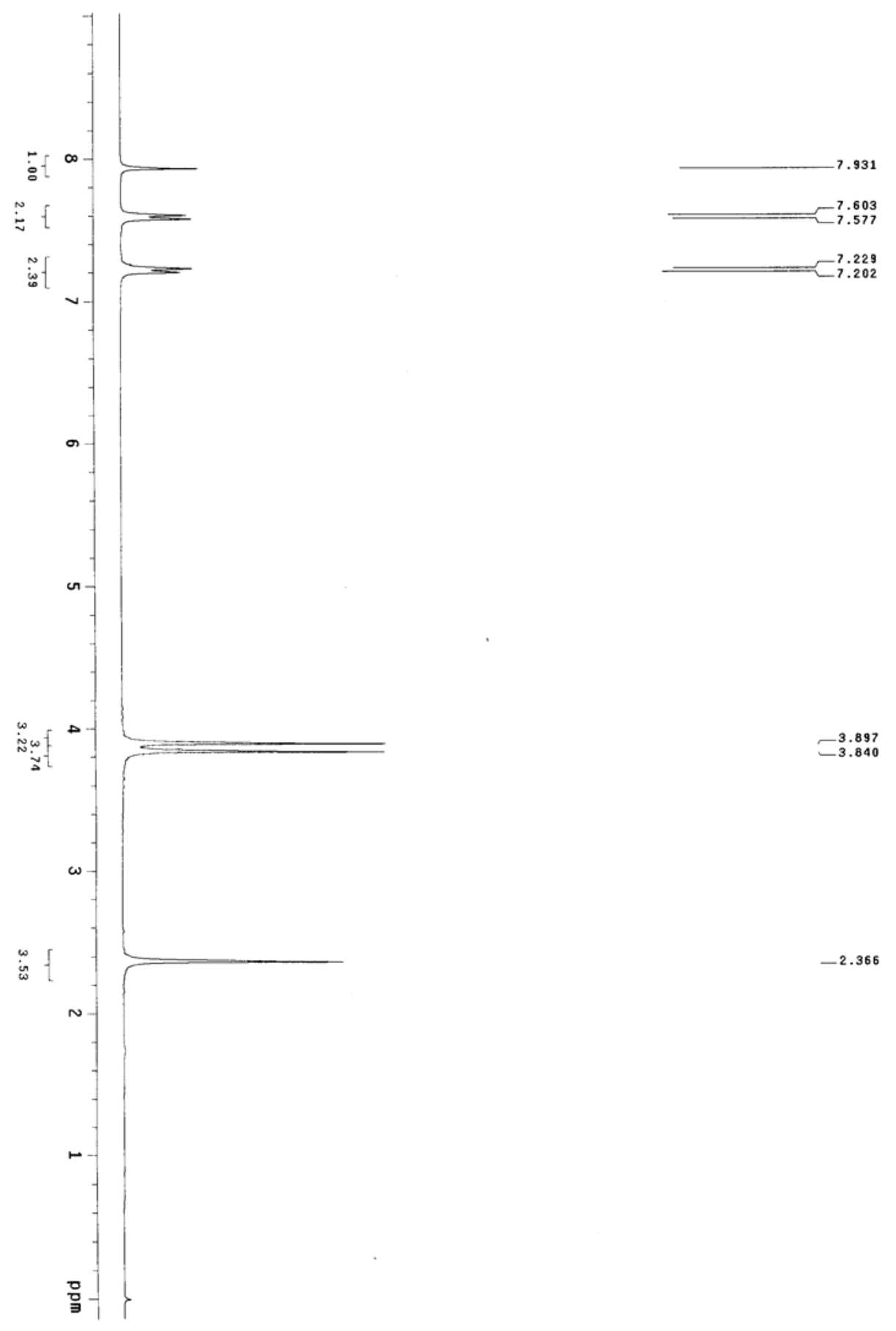

$-2.366$ 


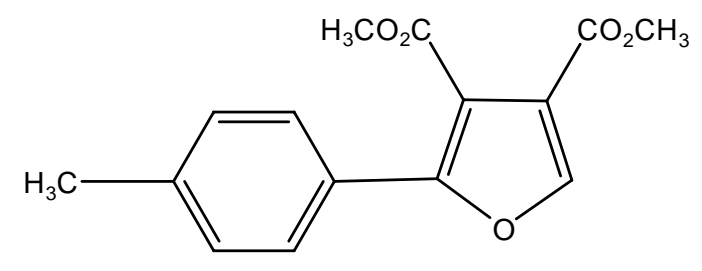



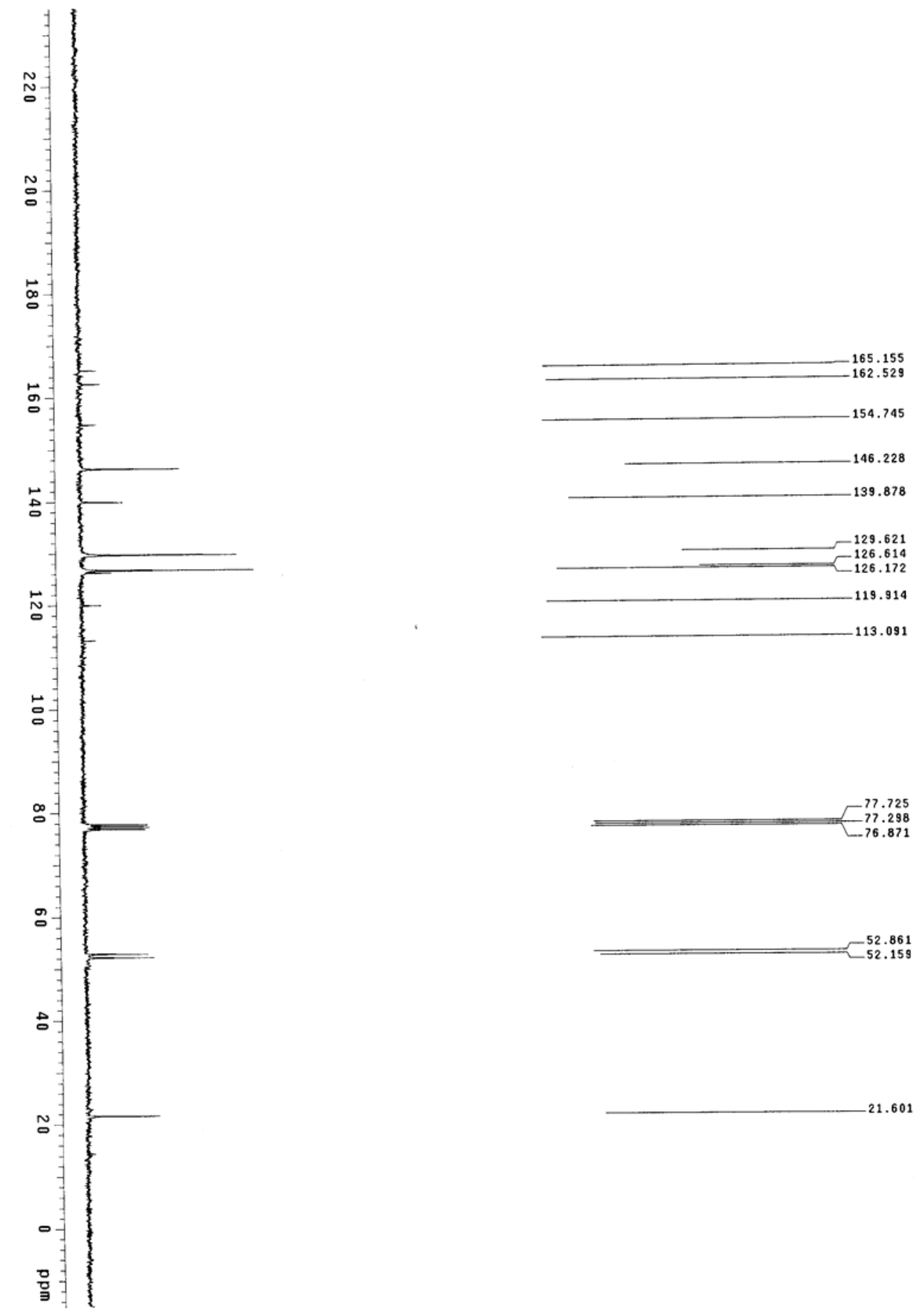


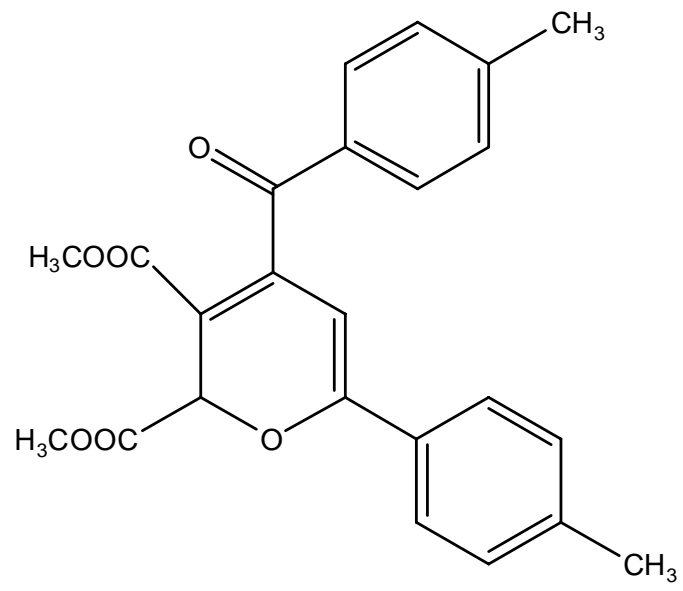

mp $148-150^{\circ} \mathrm{C}$

${ }^{1} \mathbf{H}$ NMR $\left(300 \mathrm{MHz}, \mathrm{CDCl}_{3}\right) \delta=2.38(\mathrm{~s}, 3 \mathrm{H}), 2.41(\mathrm{~s}, 3 \mathrm{H}), 3.55(\mathrm{~s}, 3 \mathrm{H}), 3.79(\mathrm{~s}, 3 \mathrm{H}), 5.95(\mathrm{~s}, 1 \mathrm{H}), 6.07(\mathrm{~s}$, $1 \mathrm{H}), 7.21(\mathrm{~d}, \mathrm{~J}=8.1 \mathrm{~Hz}, 2 \mathrm{H}), 7.21(\mathrm{~d}, \mathrm{~J}=7.8 \mathrm{~Hz}, 4 \mathrm{H}), 7.68(\mathrm{~d}, \mathrm{~J}=8.1 \mathrm{~Hz}, 2 \mathrm{H}), 7.83(\mathrm{~d}, \mathrm{~J}=8.1 \mathrm{~Hz}, 2 \mathrm{H})$.

${ }^{13} \mathrm{C}$ NMR $\left(75 \mathrm{MHz}, \mathrm{CDCl}_{3}\right) \delta=21.7,22.0,52.1,53.0,72.5,97.8,110.3,127.2,129.3,129.6,129.8$, $133.1,141.9,145.0,145.7,159.7,164.3,170.2,194.8$.

IR $(\mathrm{KBr}): \mathrm{v}=2953,1750,1709,1675,1255,1087,804 \mathrm{~cm}^{-1}$.

MS: $\mathrm{m} / \mathrm{z}(\%)=406\left(\mathrm{M}^{+}, 4.13\right), 347(100), 119(56.98), 91(44.05)$.

HRMS calcd for $\mathrm{C}_{24} \mathrm{H}_{22} \mathrm{O}_{6}$ : (M+Na) 429.1306, Found: 429.1313 . 


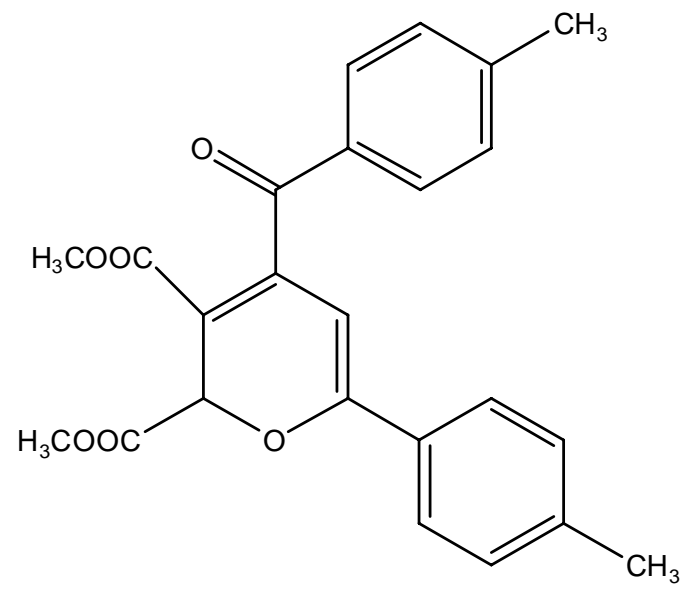



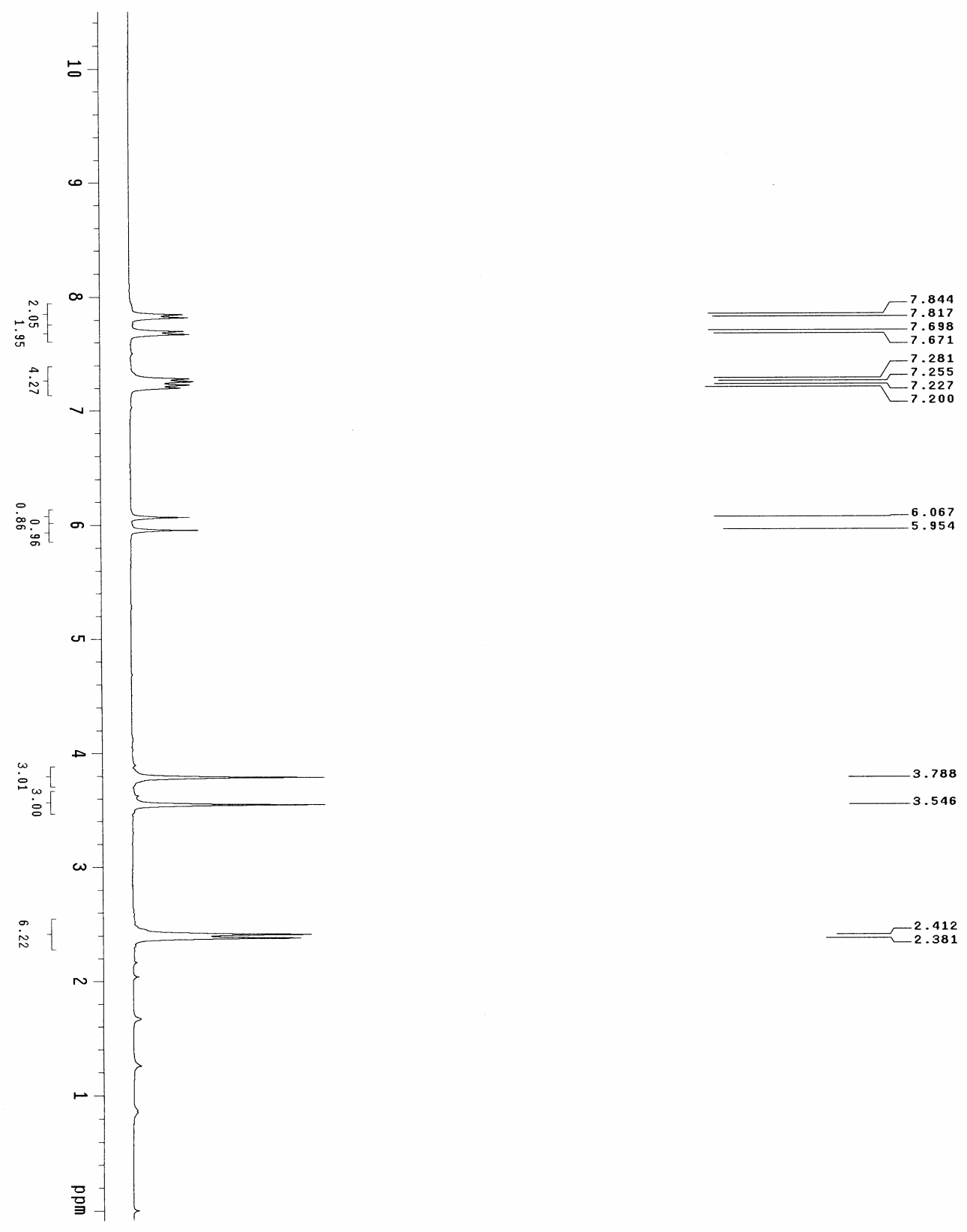


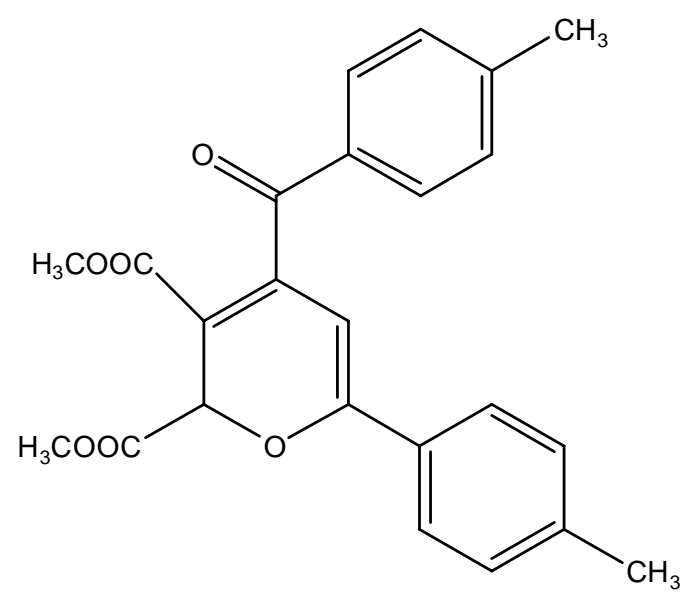



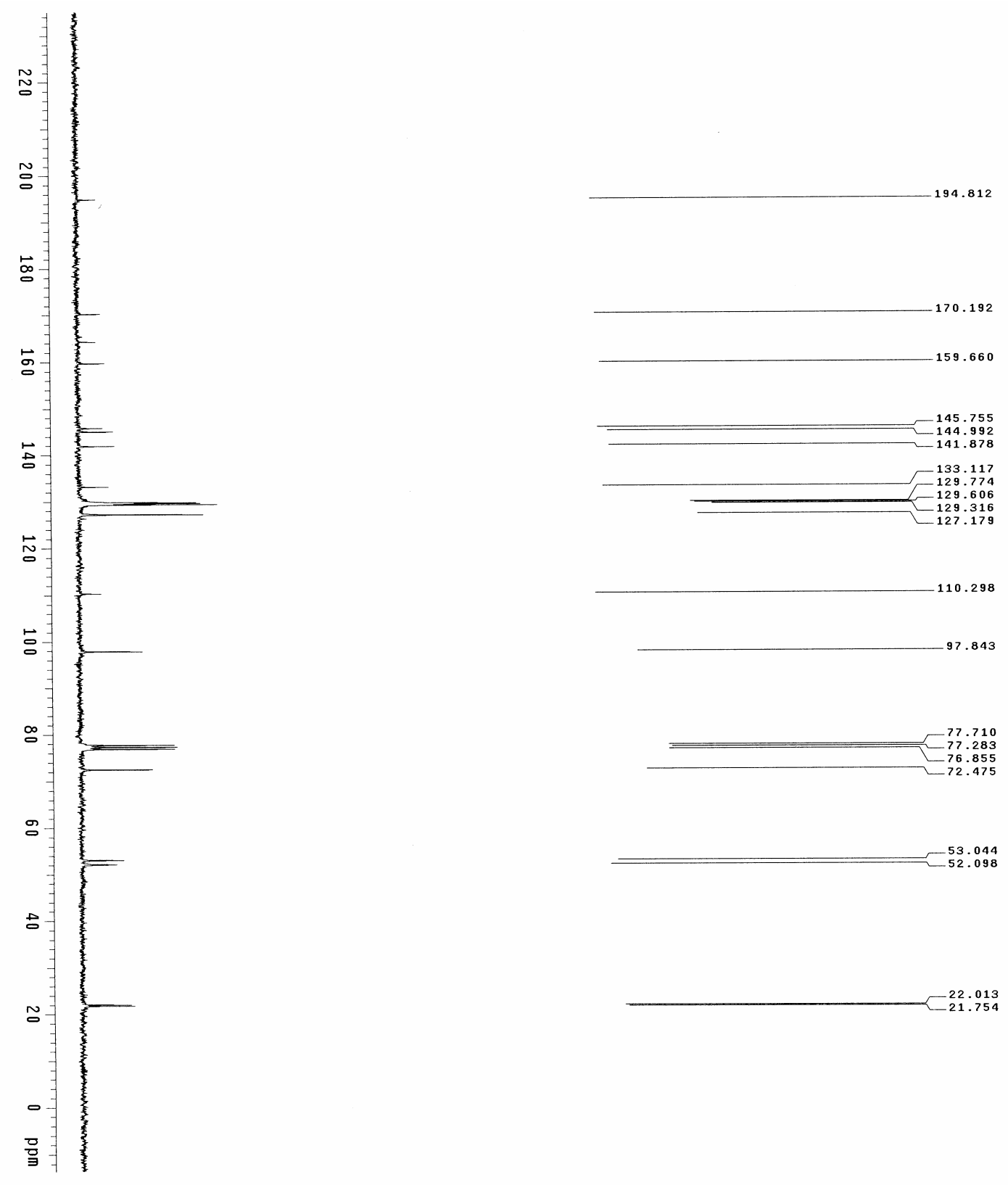

3f Dimethyl 2-(4-methoxylphenyl) furan-3, 4-dicarboxylate

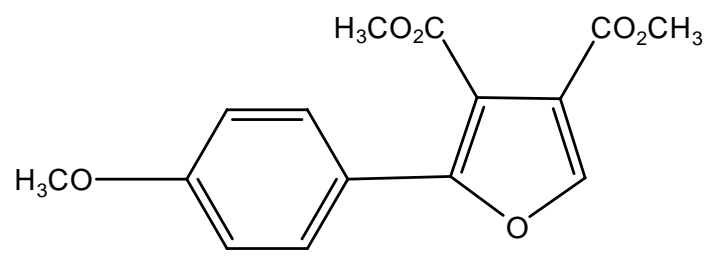

${ }^{1}$ H NMR $\left(300 \mathrm{MHz}, \mathrm{CDCl}_{3}\right) \delta=3.83(\mathrm{~s}, 3 \mathrm{H}), 3.84(\mathrm{~s}, 3 \mathrm{H}), 3.89(\mathrm{~s}, 3 \mathrm{H}), 6.94(\mathrm{~d}, \mathrm{~J}=8.4 \mathrm{~Hz}, 2 \mathrm{H}), 7.67(\mathrm{~d}$, 
$\mathrm{J}=8.7 \mathrm{~Hz}, 2 \mathrm{H}), 7.91(\mathrm{~s}, 1 \mathrm{H})$.

${ }^{13} \mathbf{C}$ NMR $\left(75 \mathrm{MHz}, \mathrm{CDCl}_{3}\right) \delta=52.1,52.8,55.5,112.3,114.3,120.0,121.6,128.4,145.9,155.0,1160.8$, 162.6, 165.1.

IR $(\mathrm{KBr}): \mathrm{v}=2953,2919,1728,1506,1256,1165,1066,733 \mathrm{~cm}^{-1}$.

MS: $\mathrm{m} / \mathrm{z}(\%)=290\left(\mathrm{M}^{+}, 100\right), 259(58.97), 135(21.06), 57(12.74), 43(15.09)$.

HRMS calcd for $\mathrm{C}_{15} \mathrm{H}_{14} \mathrm{O}_{6}$ : (M+Na) 313.0683, Found: 313.0688 .

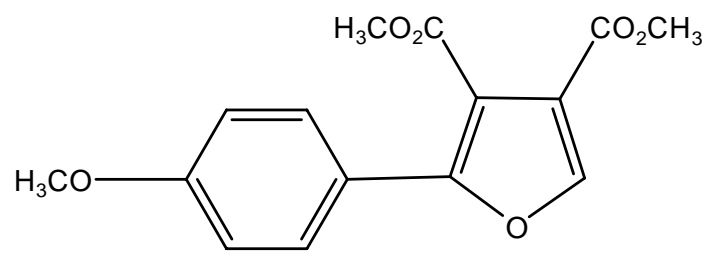



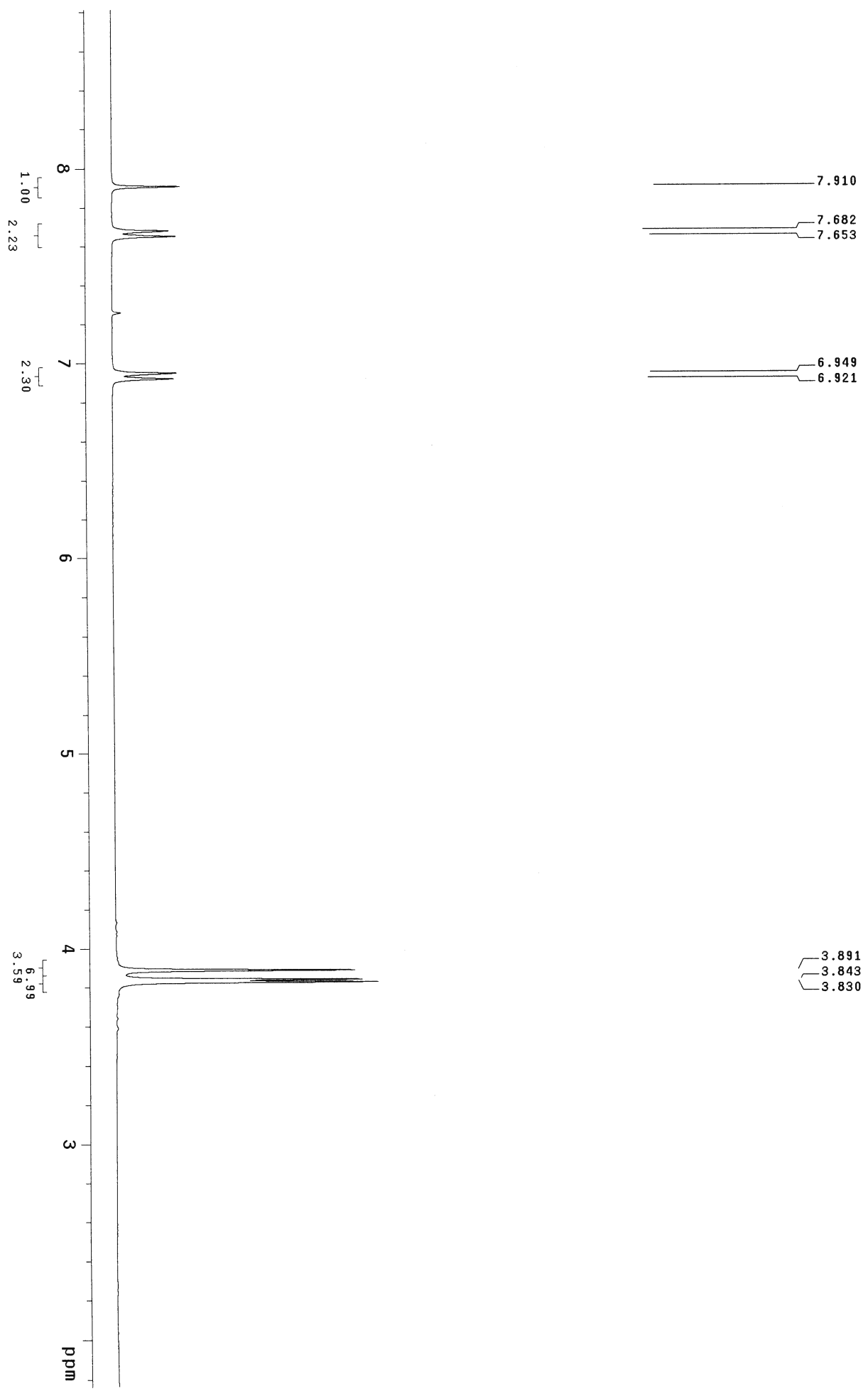

$\begin{array}{r}3.891 \\ -3.843 \\ \hline\end{array}$

$\checkmark 3.830$ 


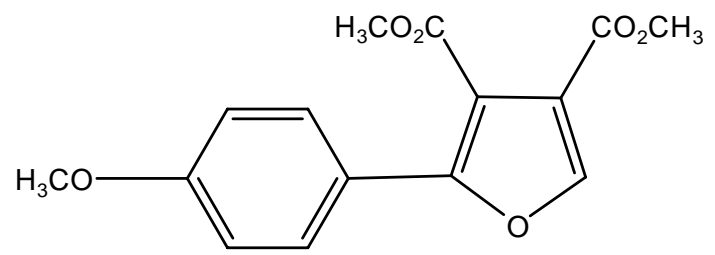



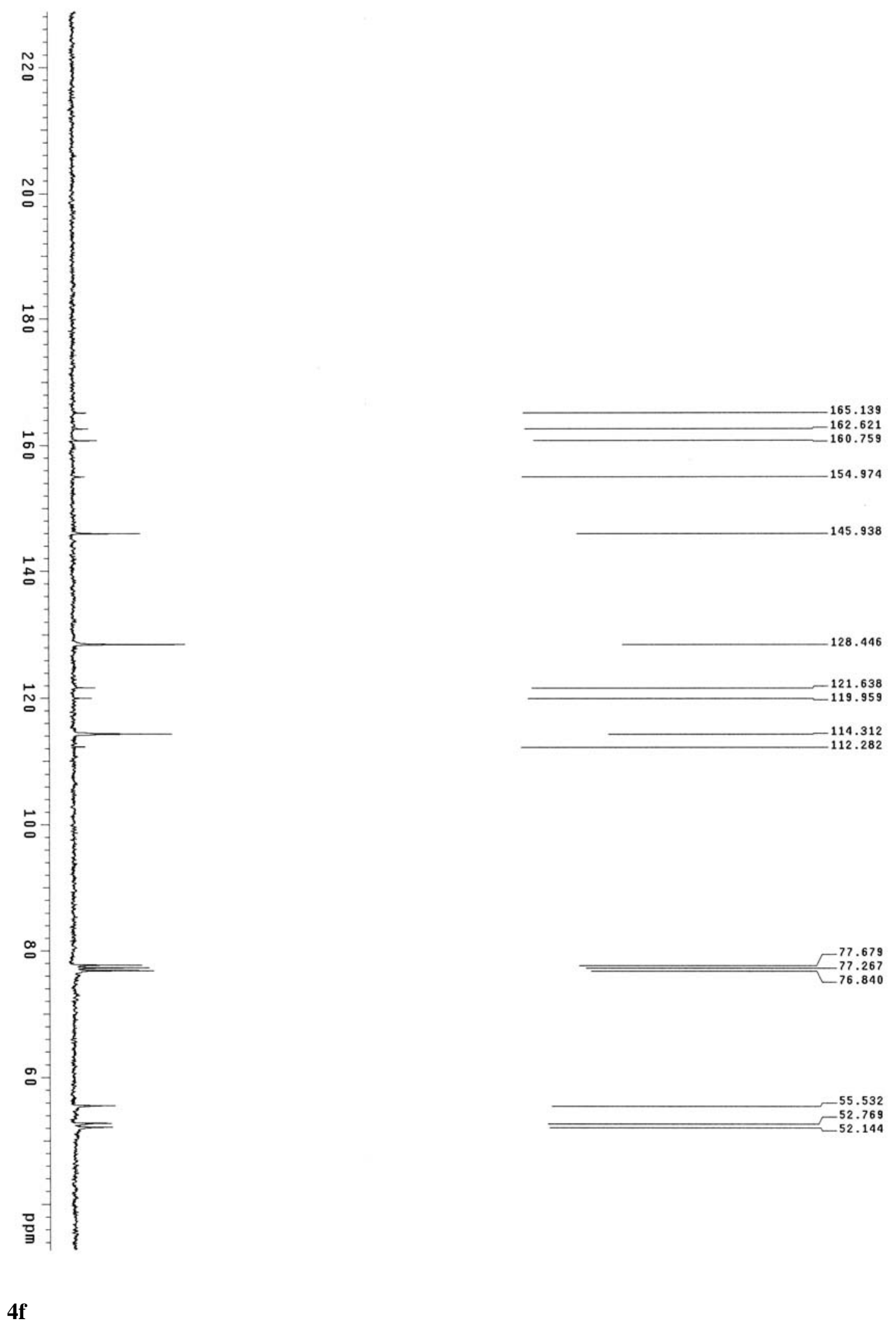


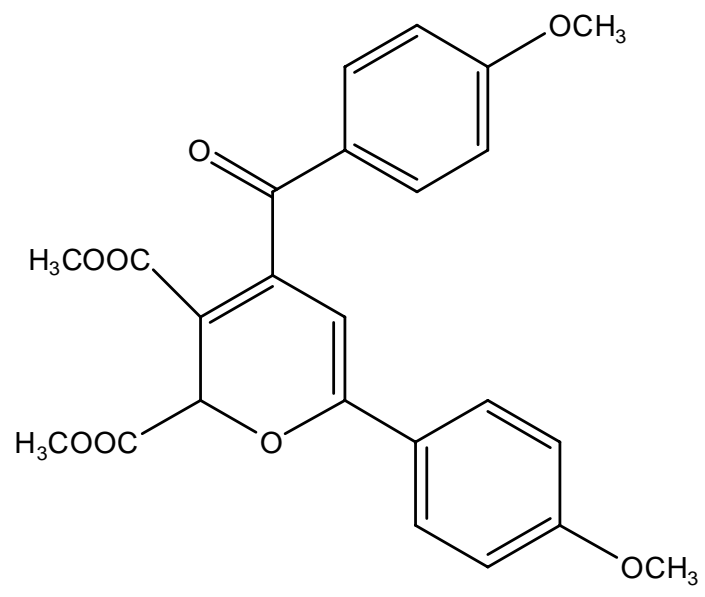

$\operatorname{mp} 132-134^{\circ} \mathrm{C}$

${ }^{1} \mathbf{H}$ NMR $\left(300 \mathrm{MHz}, \mathrm{CDCl}_{3}\right) \delta=3.54(\mathrm{~s}, 3 \mathrm{H}), 3.77(\mathrm{~s}, 3 \mathrm{H}), 3.80(\mathrm{~s}, 3 \mathrm{H}), 3.83(\mathrm{~s}, 3 \mathrm{H}), 5.88(\mathrm{~s}, 1 \mathrm{H}), 6.04(\mathrm{~s}$, 1H), 6.88-7.91(m, 8H).

${ }^{13} \mathrm{C}$ NMR $\left(75 \mathrm{MHz}, \mathrm{CDCl}_{3}\right) \delta=52.1,53.0,55.6,55.7,72.5,97.1,109.5,114.3,124.8,128.6,129.0$, 131.3, 131.6, 146.1, 159.5, 162.3, 164.3, 170.4, 193.9.

IR $(\mathrm{KBr}): \mathrm{v}=2953,1738,1708,1669,1259,1088,843 \mathrm{~cm}^{-1}$.

MS: $\mathrm{m} / \mathrm{z}(\%)=438\left(\mathrm{M}^{+}, 3.52\right), 379(67.69), 135(100)$.

HRMS calcd for $\mathrm{C}_{24} \mathrm{H}_{22} \mathrm{O}_{8}$ : (M+NH4) 456.1653, Found: 456.1647. 


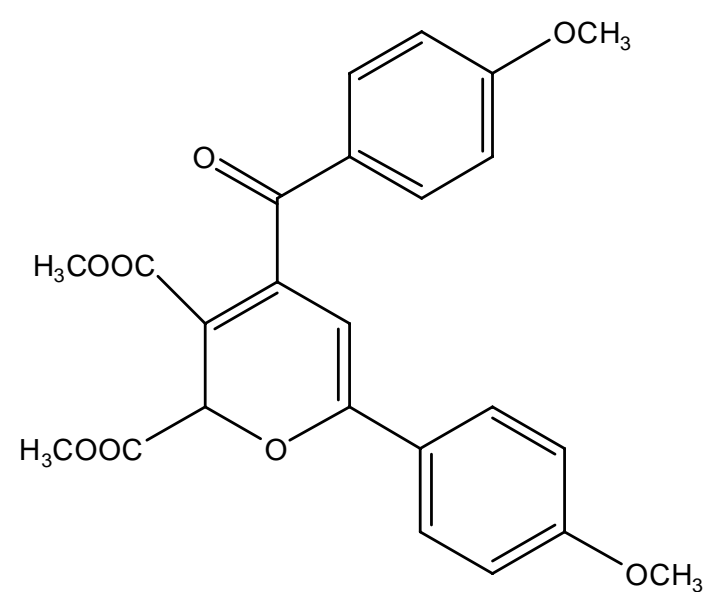



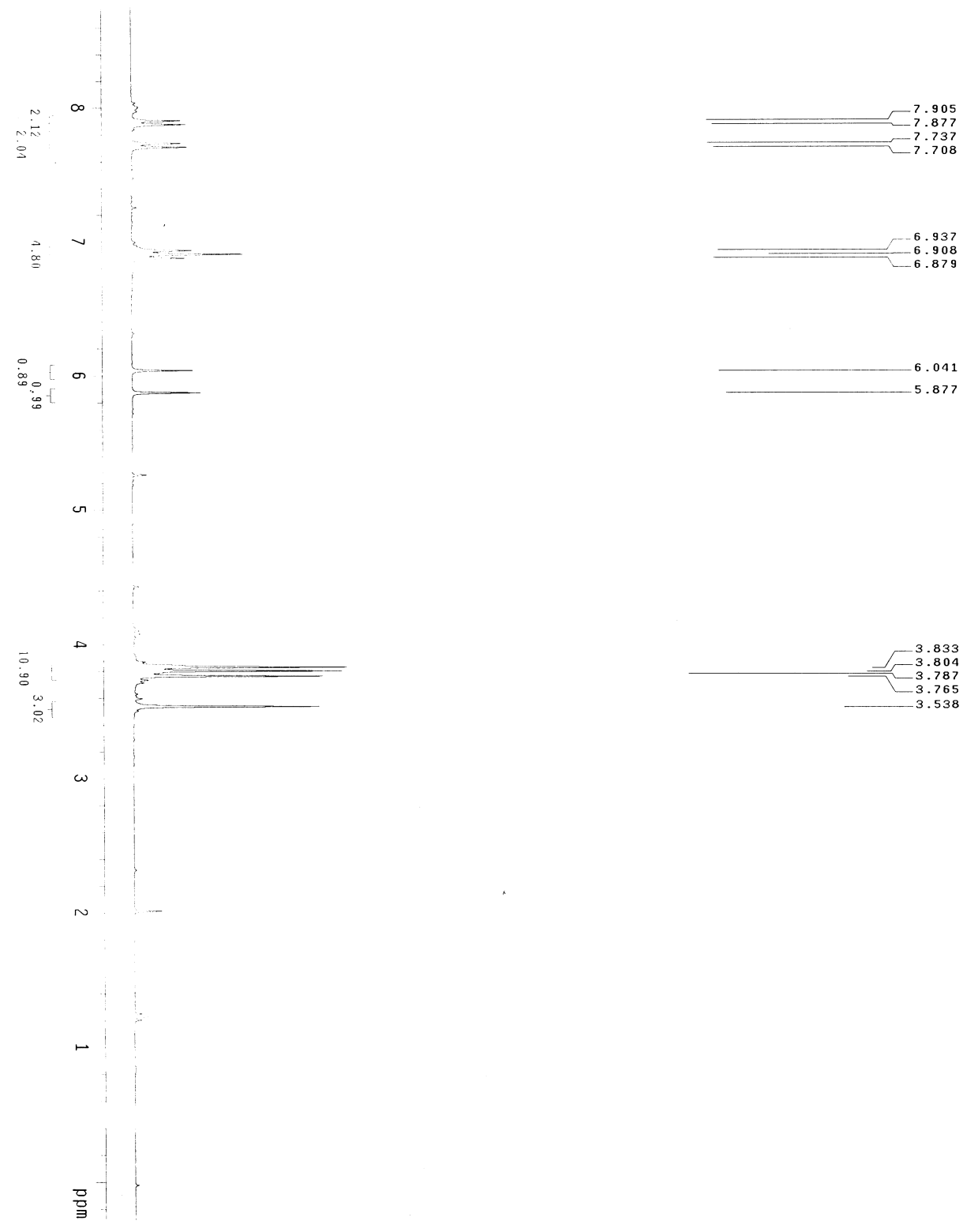


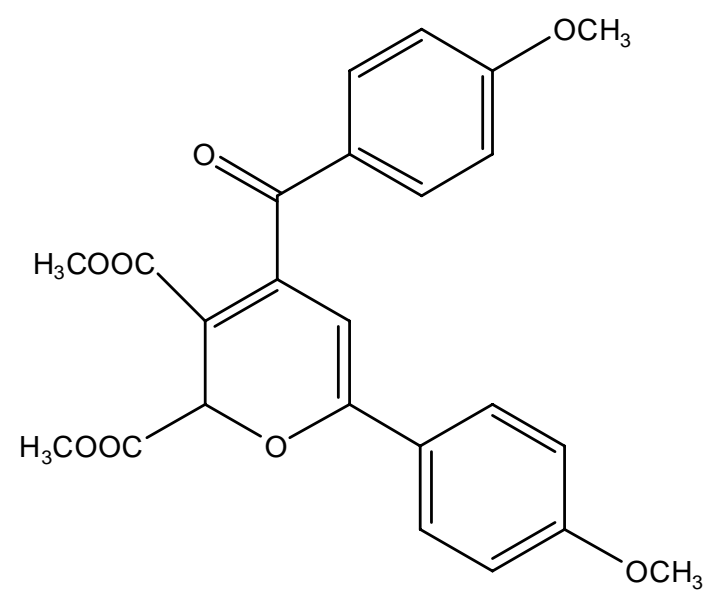



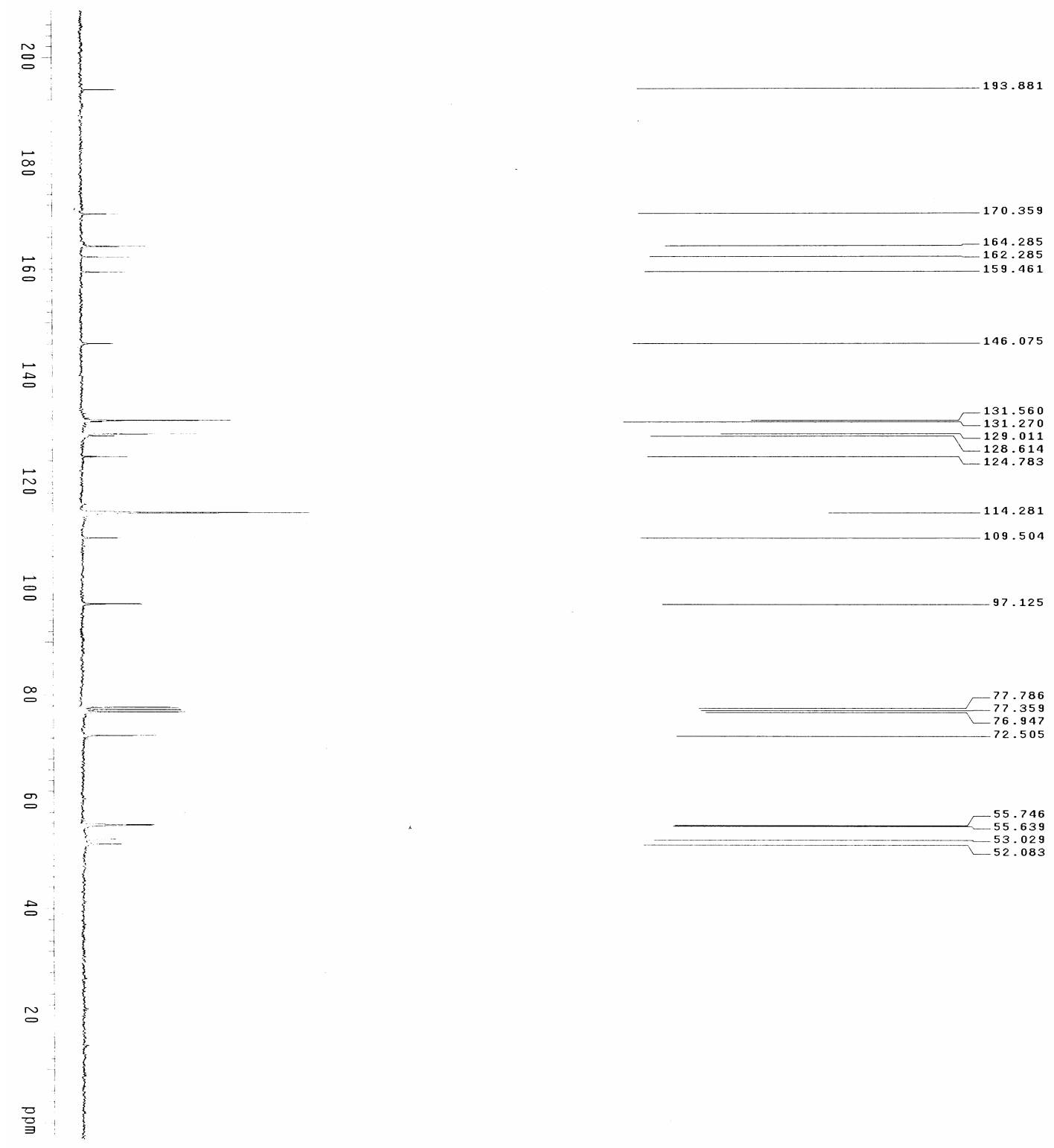

3g Dimethyl 2-(2, 4-dimethylphenyl) furan-3, 4-dicarboxylate

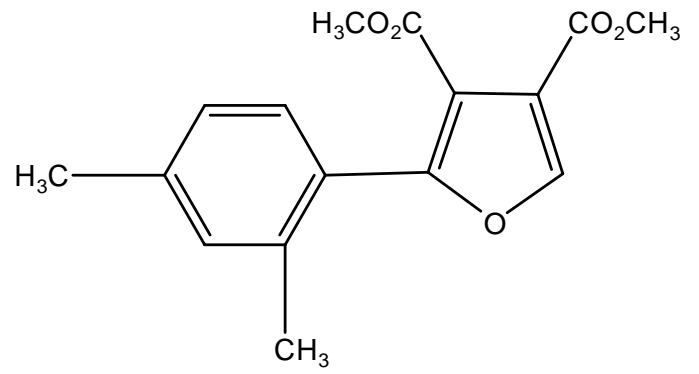


$\operatorname{mp} 50-52^{\circ} \mathrm{C}$

${ }^{1} \mathbf{H}$ NMR $\left(300 \mathrm{MHz}, \mathrm{CDCl}_{3}\right) \delta=2.25(\mathrm{~s}, 3 \mathrm{H}), 2.35(\mathrm{~s}, 3 \mathrm{H}), 3.76(\mathrm{~d}, \mathrm{~J}=2.1 \mathrm{~Hz}, 3 \mathrm{H}), 3.87(\mathrm{~d}, \mathrm{~J}=2.1 \mathrm{~Hz}, 3 \mathrm{H})$, 7.04(d, J=7.5Hz, 1H), 7.08(s, 1H), $7.28(\mathrm{~d}, \mathrm{~J}=7.8 \mathrm{~Hz}, 2 \mathrm{H}), 7.97(\mathrm{~d}, \mathrm{~J}=2.1 \mathrm{~Hz}, 1 \mathrm{H})$

${ }^{13} \mathrm{C}$ NMR $\left(75 \mathrm{MHz}, \mathrm{CDCl}_{3}\right) \delta=20.2,21.5,52.2,52.4,115.0,119.3,125.7,126.5,130.5,131.6,137.8$

$140.3,146.6,157.6,162.7,164.0$.

IR (KBr): v $=2953,1732,1066,818,764 \mathrm{~cm}^{-1}$.

MS: $\mathrm{m} / \mathrm{z}(\%)=288\left(\mathrm{M}^{+}, 6.79\right), 257(54.59) 225(39.60), 198(76.45), 133(100)$.

HRMS calcd for $\mathrm{C}_{16} \mathrm{H}_{16} \mathrm{O}_{5}$ : (M+Na) 311.0890, Found: 311.0885. 


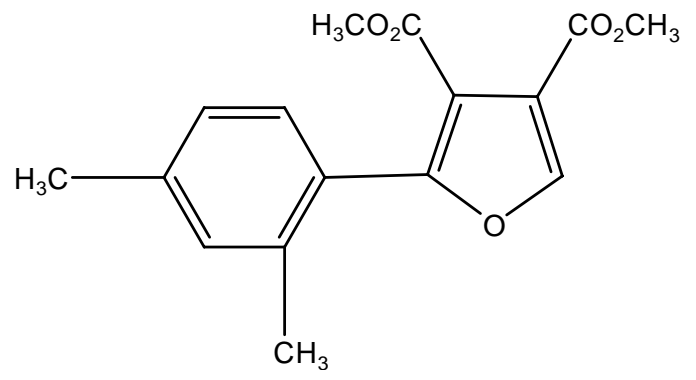



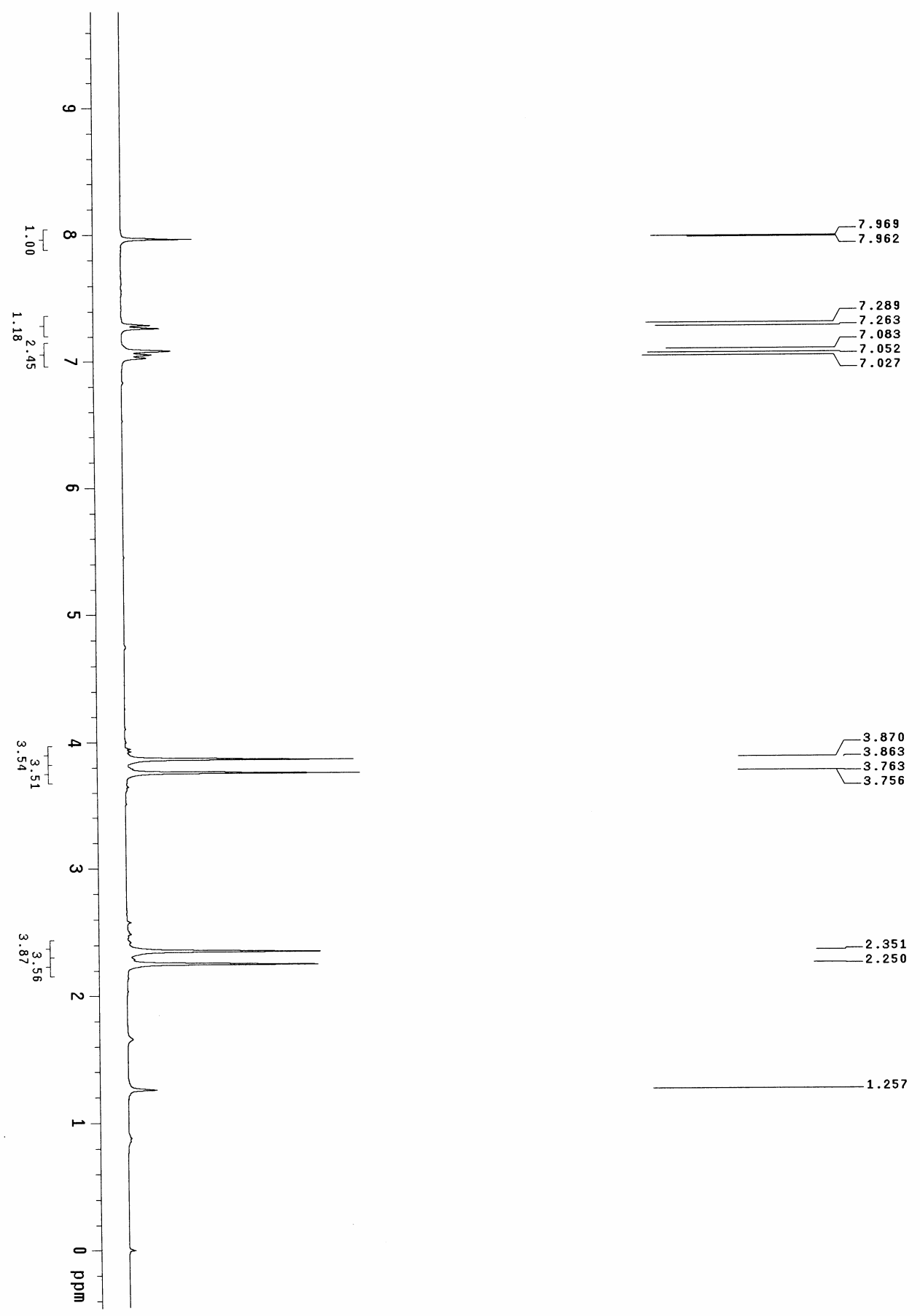


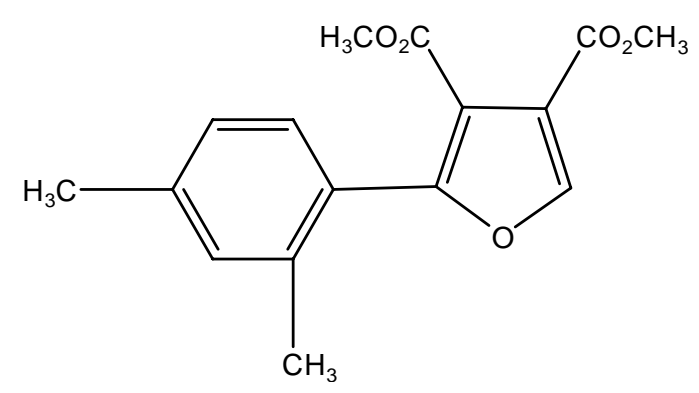



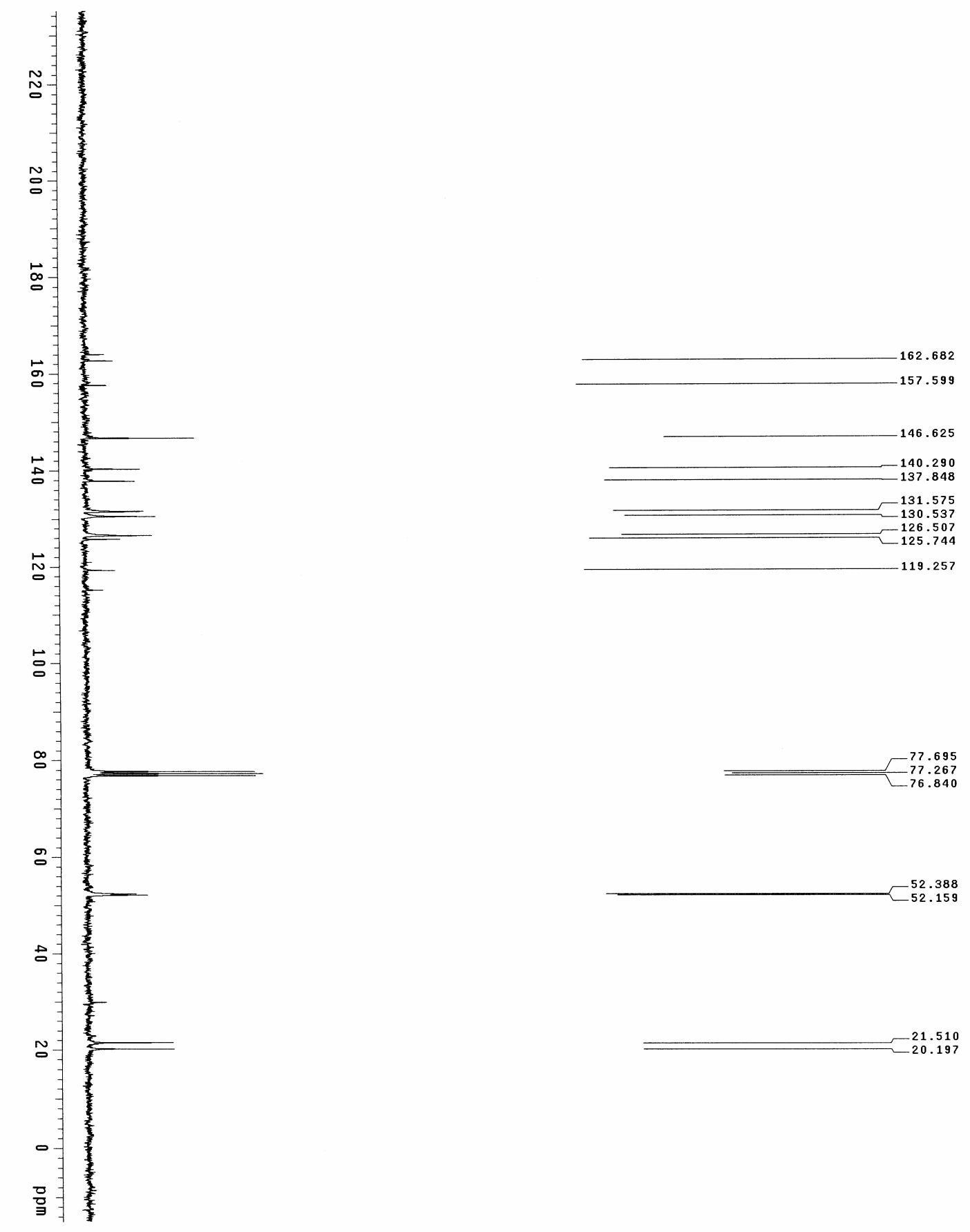

$4 g$ 


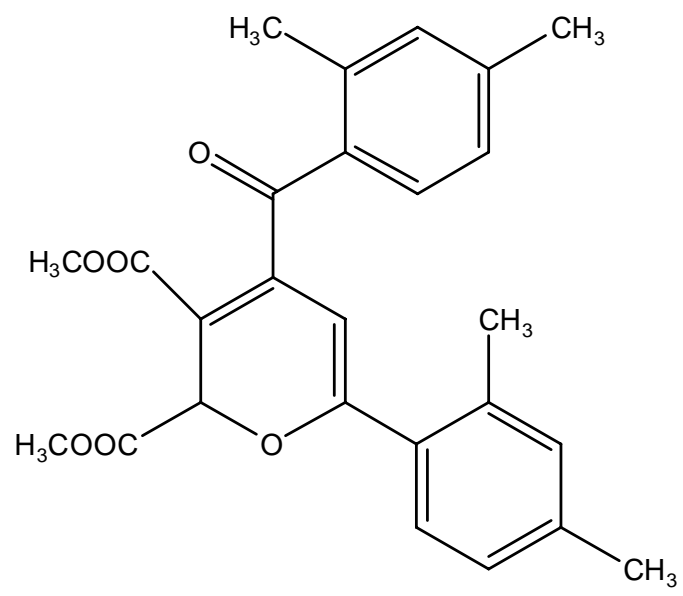

mp $103-104^{\circ} \mathrm{C}$

${ }^{1} \mathbf{H}$ NMR $\left(300 \mathrm{MHz}, \mathrm{CDCl}_{3}\right) \delta=2.34(\mathrm{~s}, 3 \mathrm{H}), 2.36(\mathrm{~s}, 3 \mathrm{H}), 2.42(\mathrm{~s}, 3 \mathrm{H}), 2.69(\mathrm{~s}, 3 \mathrm{H}), 3.55(\mathrm{~s}, 3 \mathrm{H}), 3.81(\mathrm{~s}$, $3 \mathrm{H}), 5.67(\mathrm{~s}, 1 \mathrm{H}), 6.02(\mathrm{~s}, 1 \mathrm{H}), 7.04-7.69(\mathrm{~m}, 6 \mathrm{H})$.

${ }^{13} \mathrm{C}$ NMR $\left(75 \mathrm{MHz}, \mathrm{CDCl}_{3}\right) \delta=21.1,21.2,21.4,21.7,51.8,52.6,72.3,102.3,108.5,126.3,126.7$, $129.4,130.1,131.7,131.9,133.1,136.7,140.4,140.5,143.3,146.4,159.8,164.0,169.7,196.0$. IR $(\mathrm{KBr}): \mathrm{v}=2954,1748,1710,1671,1248,1075,814,776 \mathrm{~cm}^{-1}$.

MS: $\mathrm{m} / \mathrm{z}(\%)=434\left(\mathrm{M}^{+}, 2.35\right), 375(49.43), 133(100), 105(34.95)$.

HRMS calcd for $\mathrm{C}_{26} \mathrm{H}_{26} \mathrm{O}_{6}$ : (M+H) 435.1802, Found: 435.1799 . 


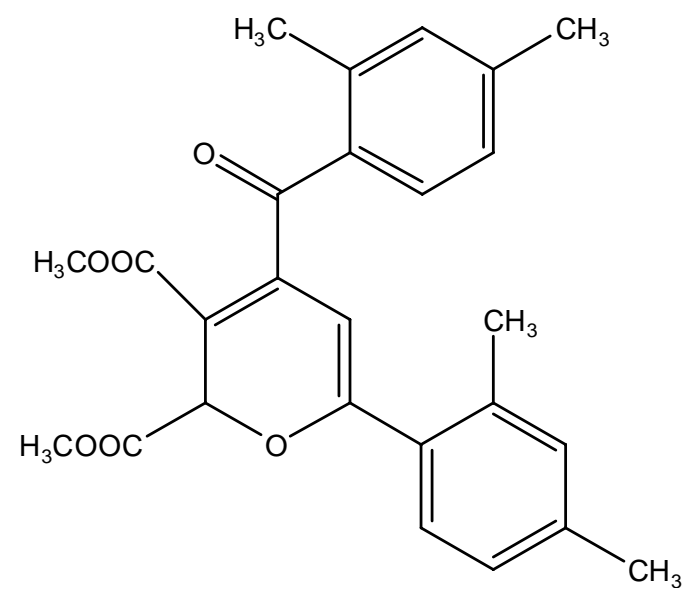



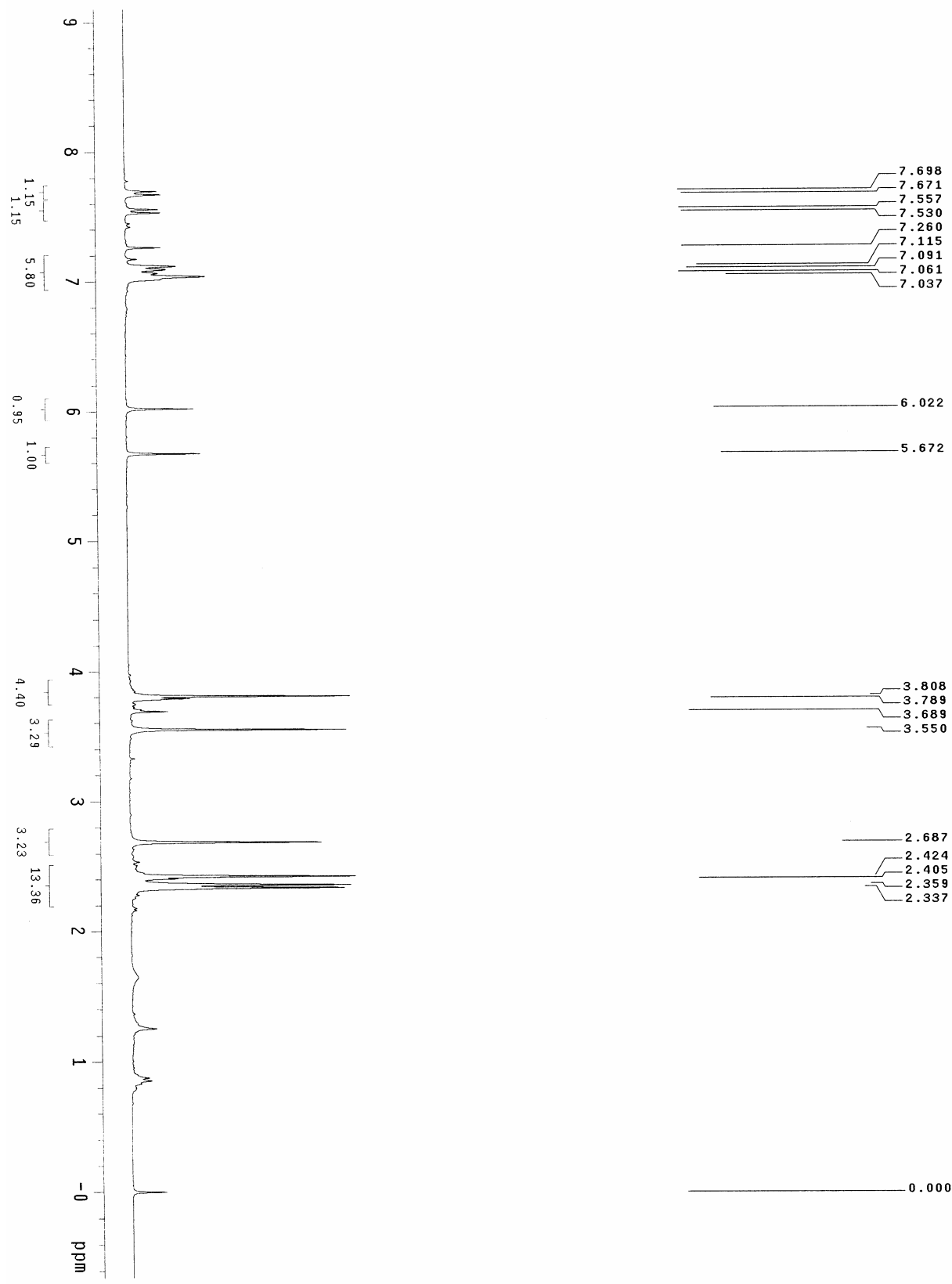


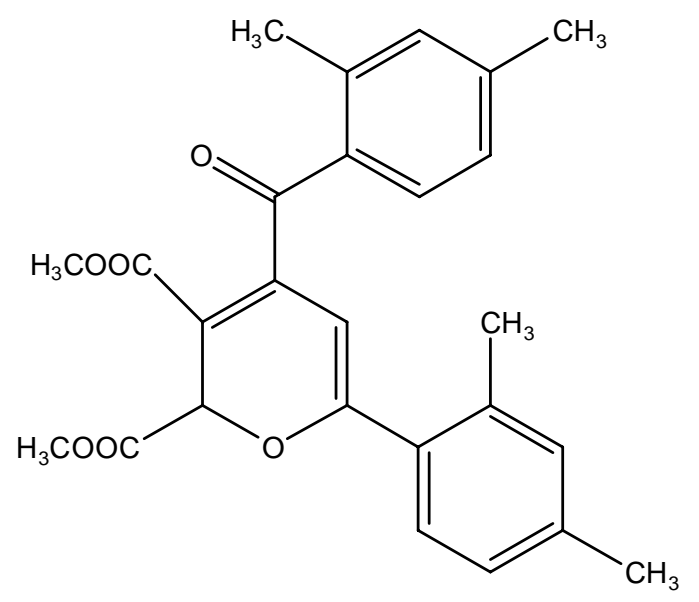



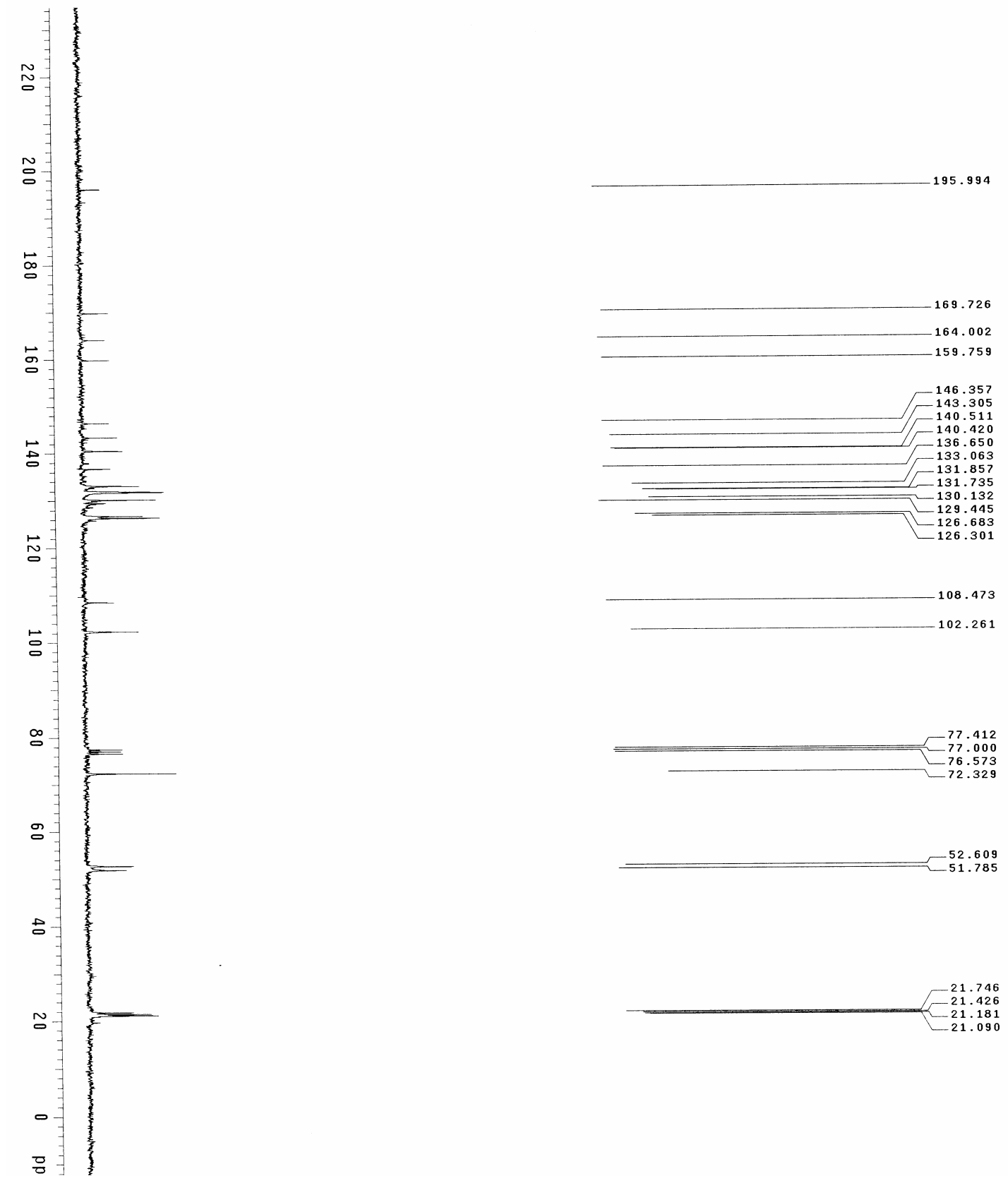

3h Dimethyl 2-(1,2,3,4-tetrahydronaphthalen-5-yl) furan-3, 4-dicarboxylate 


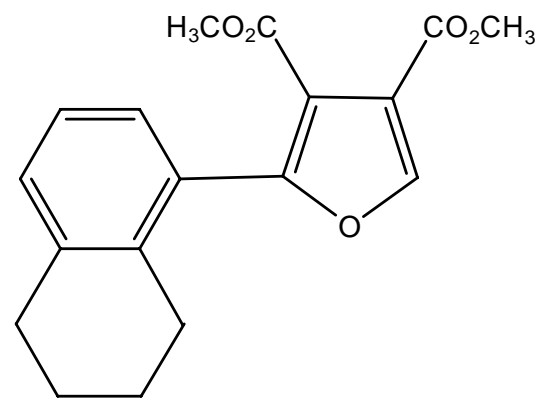

$\operatorname{mp} 88-90^{\circ} \mathrm{C}$

${ }^{1} \mathbf{H}$ NMR $\left(300 \mathrm{MHz}, \mathrm{CDCl}_{3}\right) \delta=1.80(\mathrm{~s}, 4 \mathrm{H}), 2.79(\mathrm{~s}, 4 \mathrm{H}), 3.85(\mathrm{~s}, 3 \mathrm{H}), 3.90(\mathrm{~s}, 3 \mathrm{H}), 7.09-7.41(\mathrm{~m}, 3 \mathrm{H})$, 7.93(s, 1H).

${ }^{13} \mathrm{C}$ NMR $\left(75 \mathrm{MHz}, \mathrm{CDCl}_{3}\right) \delta=23.0,29.3,29.4,51.9,52.6,112.7,119.6,123.5,125.8,127.1,129.4$, $137.5,139.0,145.9,154.7,162.3,164.9$.

IR (KBr): v=2936, 1732, 1065, 813, 733 $\mathrm{cm}^{-1}$.

MS: $\mathrm{m} / \mathrm{z}(\%)=314\left(\mathrm{M}^{+}, 100\right), 283(43.69), 196(54.83)$.

HRMS calcd for $\mathrm{C}_{18} \mathrm{H}_{18} \mathrm{O}_{5}$ : (M+Na) 337.1046, Found: 337.1038. 


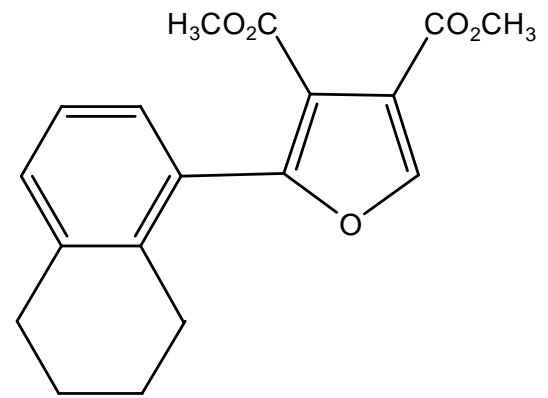



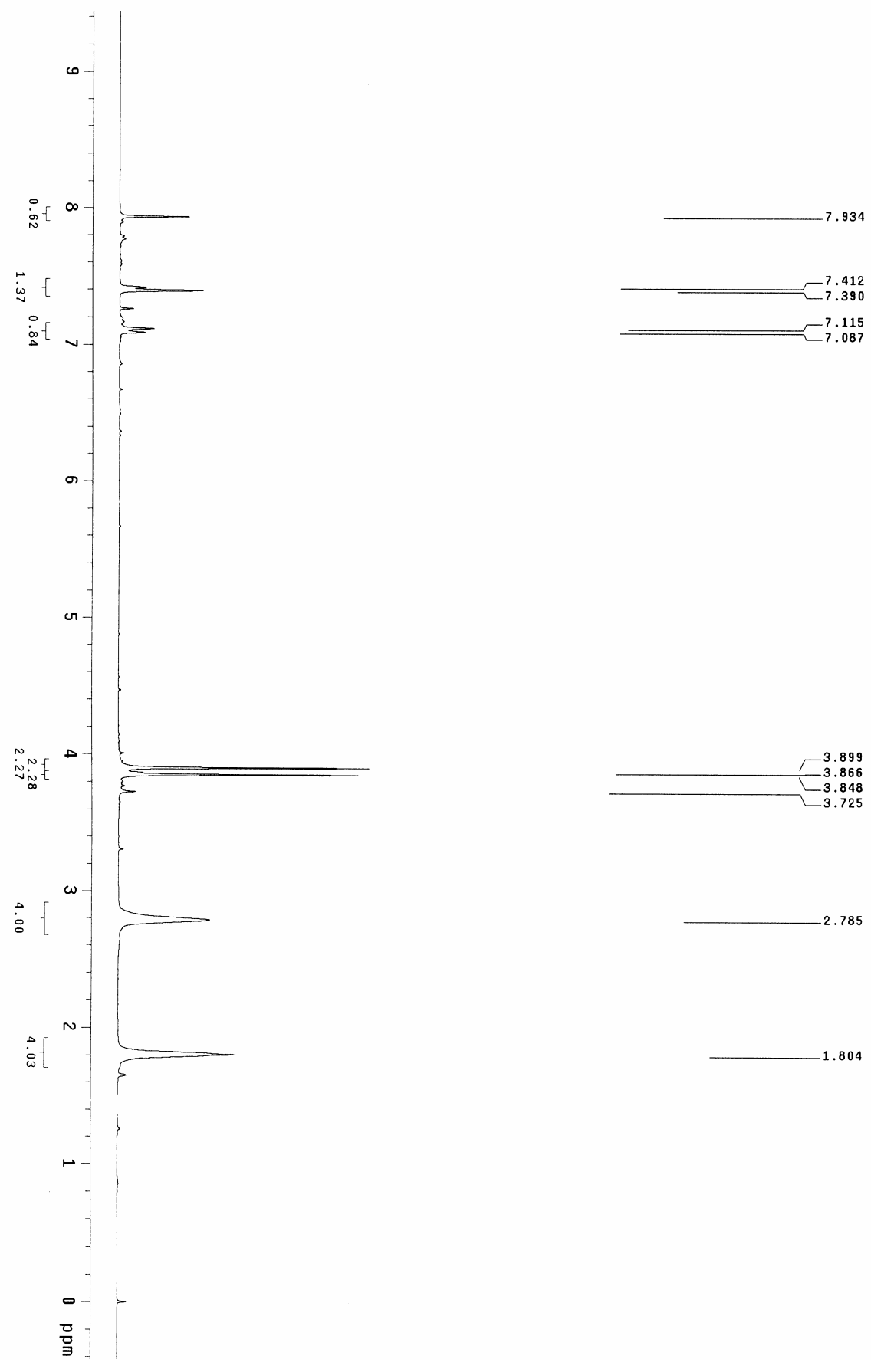


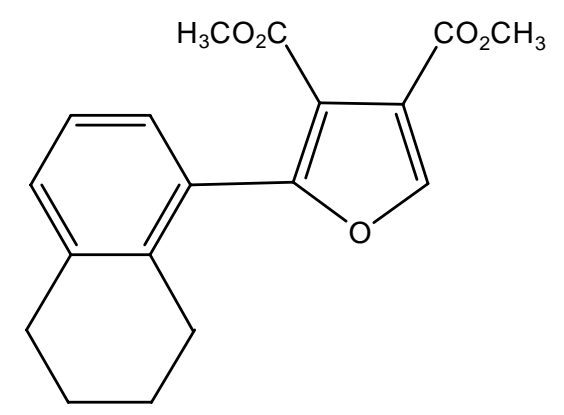



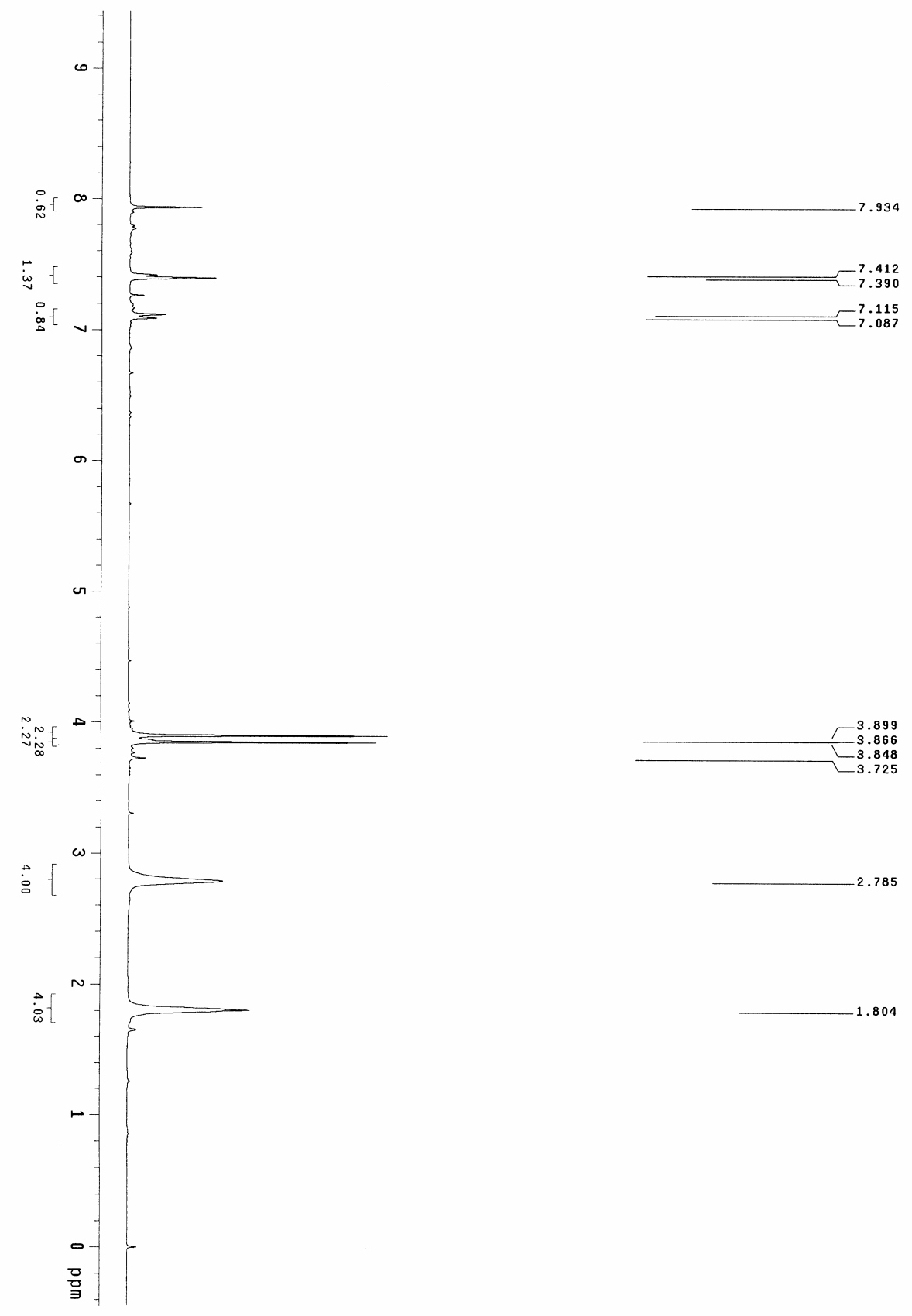

$4 h$ 


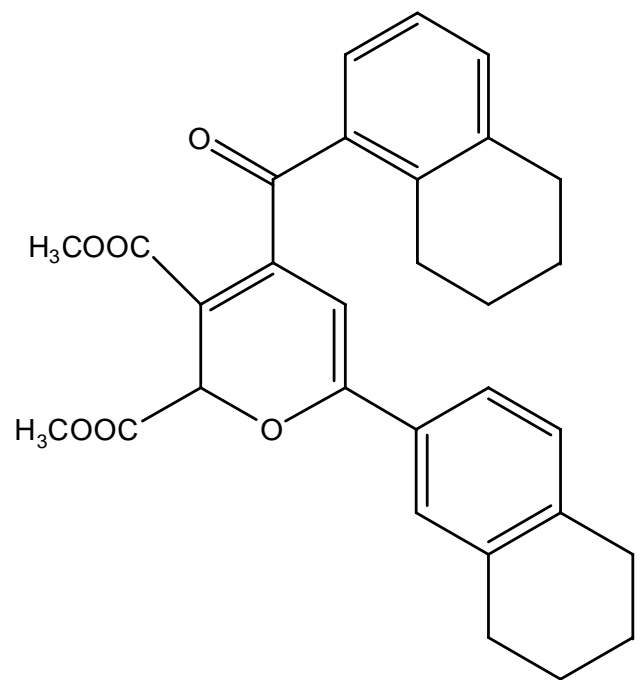

${ }^{1} \mathbf{H}$ NMR $\left(300 \mathrm{MHz}, \mathrm{CDCl}_{3}\right) \delta=1.79-1.81(\mathrm{~m}, 8 \mathrm{H}), 2.79(\mathrm{~m}, 8 \mathrm{H}), 3.59(\mathrm{~d}, \mathrm{~J}=2.4 \mathrm{~Hz}, 3 \mathrm{H}), 3.80(\mathrm{~d}, \mathrm{~J}=2.4 \mathrm{~Hz}$, 3H) $5.93(\mathrm{~d}, \mathrm{~J}=2.4 \mathrm{~Hz}, 1 \mathrm{H}), 6.06(\mathrm{~d}, \mathrm{~J}=2.4 \mathrm{~Hz}, 1 \mathrm{H}), 7.08-7.64(\mathrm{~m}, 6 \mathrm{H})$.

${ }^{13} \mathrm{C}$ NMR $\left(75 \mathrm{MHz}, \mathrm{CDCl}_{3}\right) \delta=22.7,22.8,22.9$ 29.3, 29.5, 29.7, 51.9, 52.7, 72.2, 97.6, 109.6, 124.0, $126.1,127.6,129.2,129.4,129.6,129.8,132.7,137.5,137.7,141.0,144.1,145.9,159.6,164.1,170.0$, 194.9.

IR $(\mathrm{KBr}): \mathrm{v}=2933,1741,1709,1674,1257,1080,733 \mathrm{~cm}^{-1}$.

MS: $\mathrm{m} / \mathrm{z}(\%)=486\left(\mathrm{M}^{+}, 0.99\right), 427(18.08), 159(100), 131(19.65)$.

HRMS calcd for $\mathrm{C}_{30} \mathrm{H}_{30} \mathrm{O}_{6}$ : (M+Na) 509.1935, Found: 509.1941. 


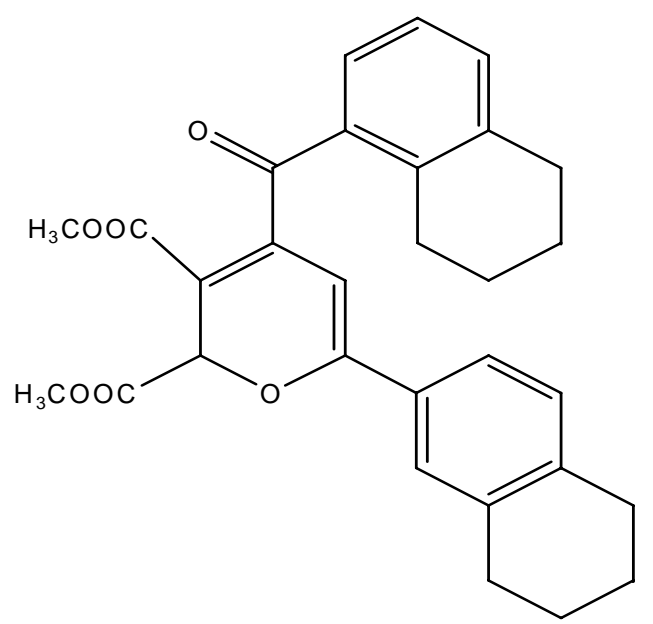



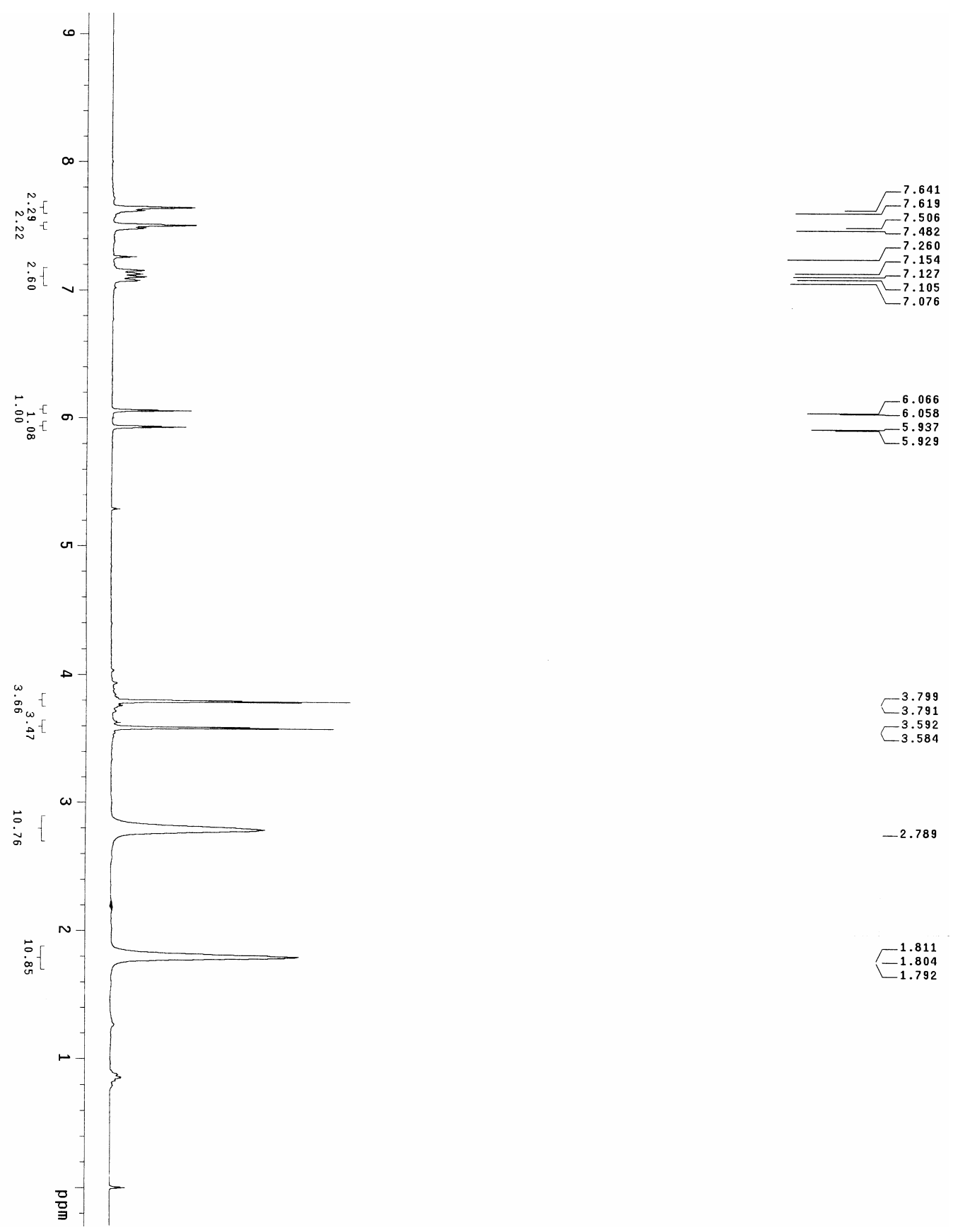

-3.799
-3.791
-3.592

-3.791
-3.584

$-2.789$

1.811
-1.804
-1.792

-1.804
-1.792 


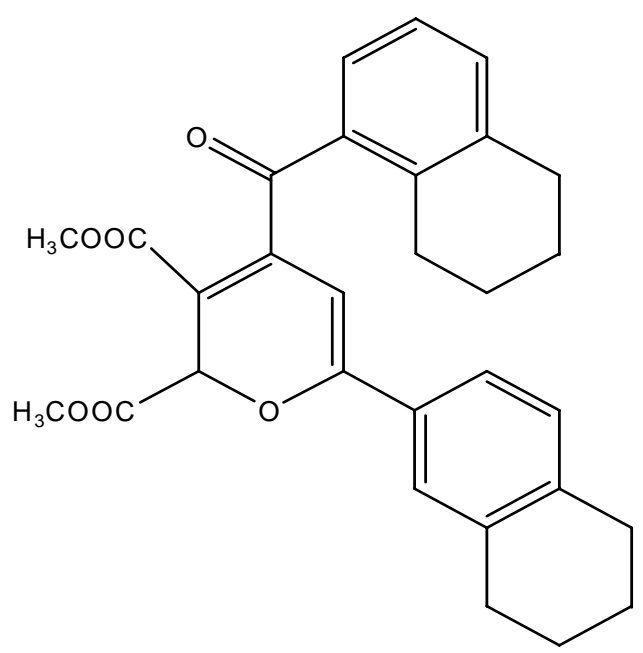



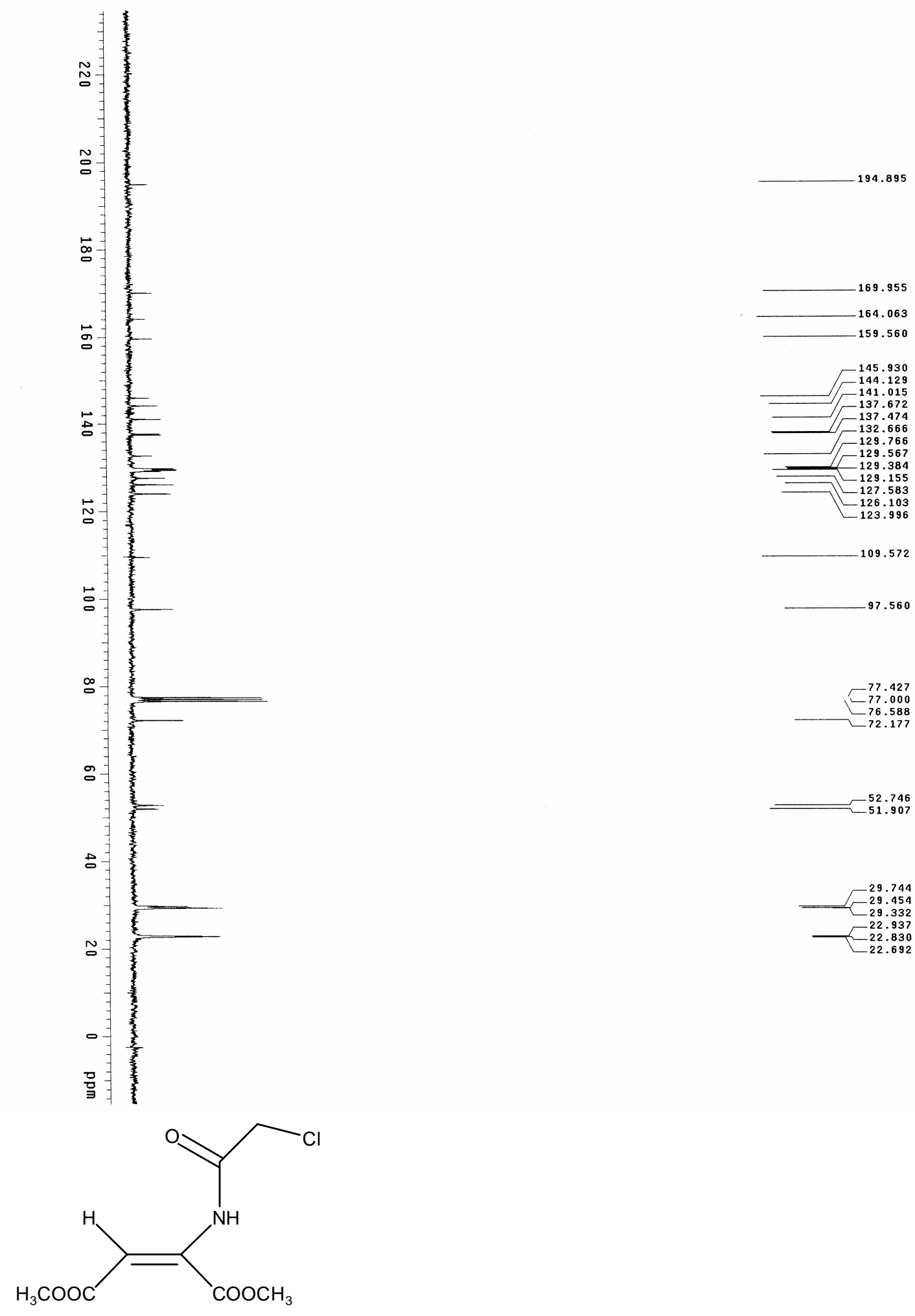

${ }^{1} \mathrm{H}$ NMR $\left(300 \mathrm{MHz}, \mathrm{CDCl}_{3}\right) \delta=3.77(\mathrm{~s}, 3 \mathrm{H}), 3.83(\mathrm{~s}, 3 \mathrm{H}), 4.14(\mathrm{~s}, 2 \mathrm{H}), 5.64(\mathrm{~s}, 1 \mathrm{H}), 11.03(\mathrm{~s}, 1 \mathrm{H})$,

${ }^{13} \mathrm{C}$ NMR $\left(75 \mathrm{MHz}, \mathrm{CDCl}_{3}\right) \delta=42.4,52.4,53.4,104.3,142.4,163.9,164.8,168.0$. 
MS: $\mathrm{m} / \mathrm{z}(\%)=235\left(\mathrm{M}^{+}, 5.36\right), 237(1.85), 204(11.46), 206(3.76), 176(100), 178(30.74)$.

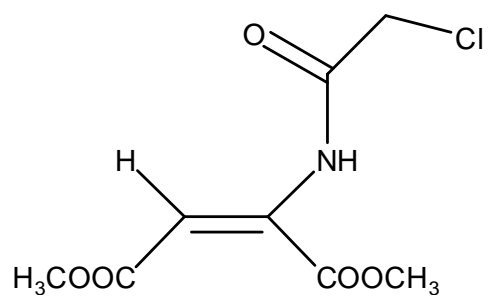




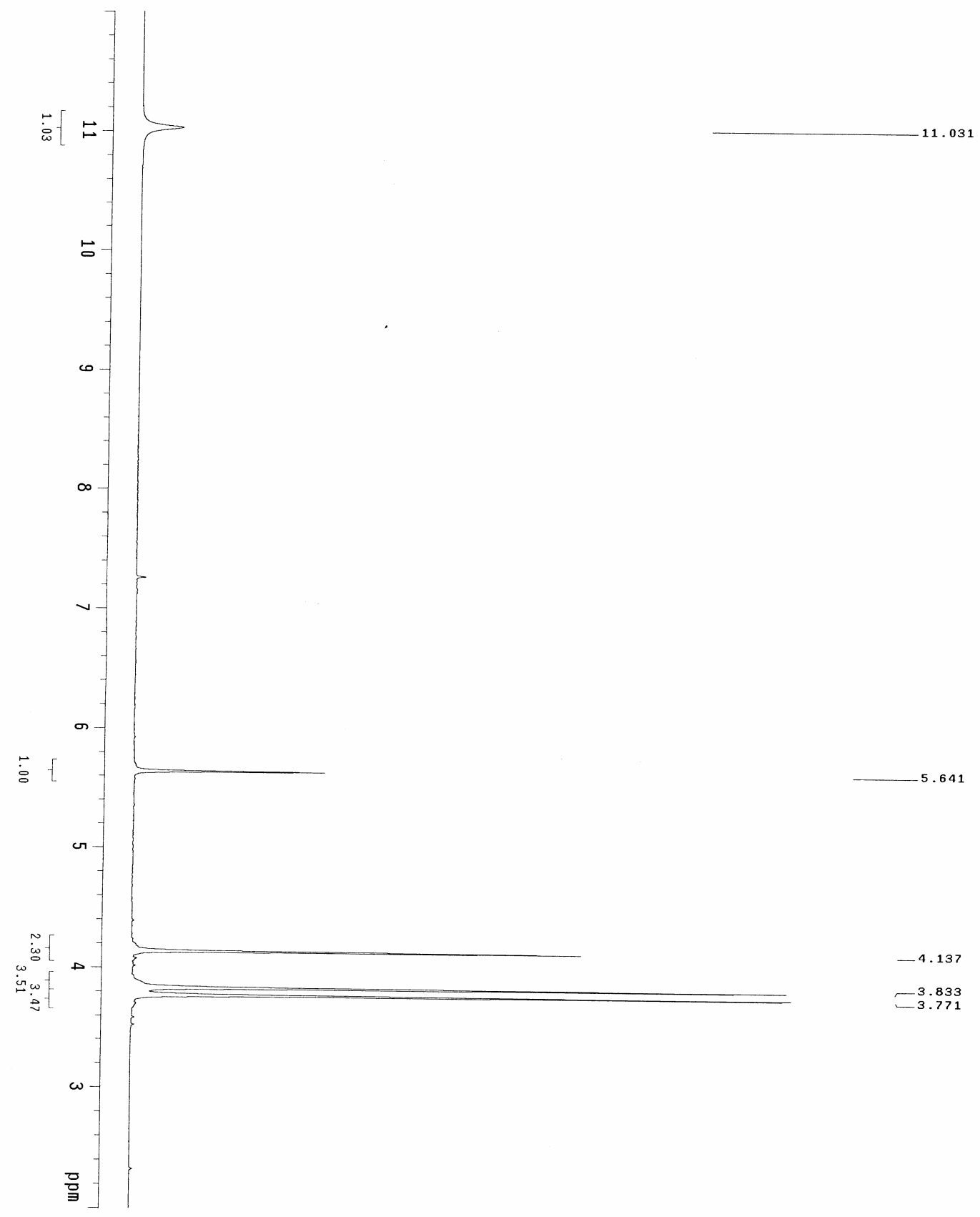




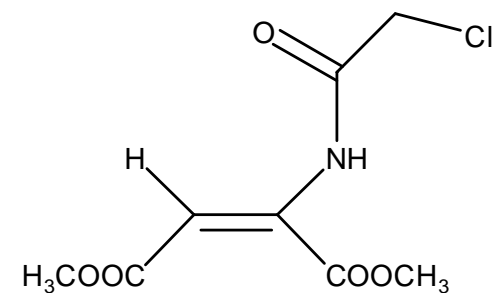



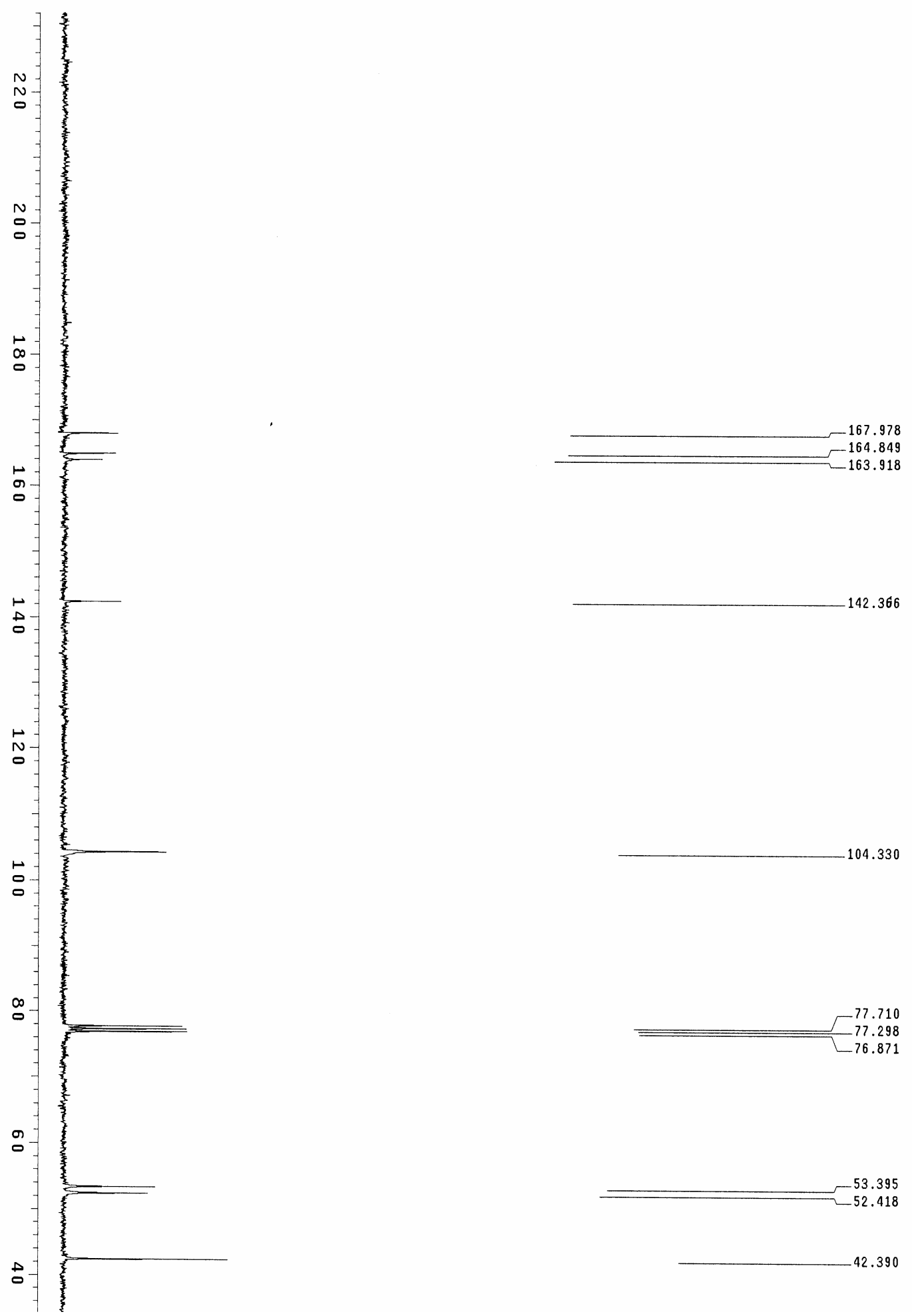

$-142.366$

$-104.330$
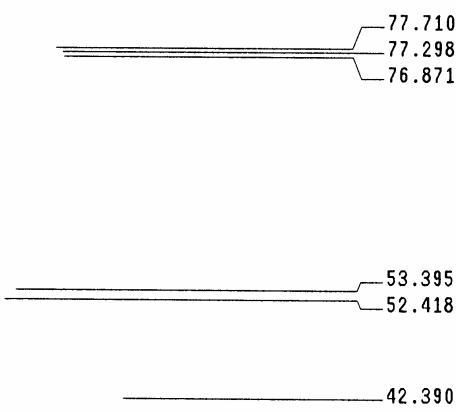


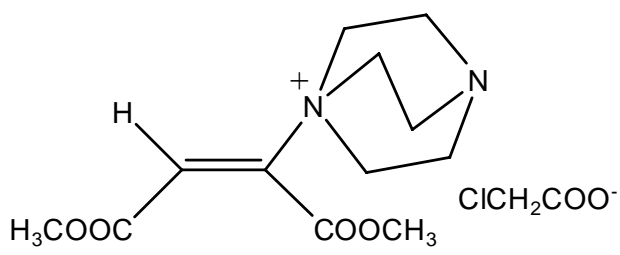

${ }^{1} \mathbf{H}$ NMR $\left(300 \mathrm{MHz}, \mathrm{CDCl}_{3}\right) \delta=2.55(\mathrm{dd}, \mathrm{J}=5.1 \mathrm{~Hz}, \mathrm{~J}=4.8 \mathrm{~Hz}, 4 \mathrm{H}), 2.66(\mathrm{dd}, \mathrm{J}=5.7 \mathrm{~Hz}, \mathrm{~J}=5.4 \mathrm{~Hz}, 2 \mathrm{H})$, $3.15(\mathrm{dd}, \mathrm{J}=5.1 \mathrm{~Hz}, \mathrm{~J}=4.8 \mathrm{~Hz}, 4 \mathrm{H}), 3.62(\mathrm{~s}, 3 \mathrm{H}), 3.90(\mathrm{~s}, 3 \mathrm{H}), 4.06(\mathrm{~s}, 2 \mathrm{H}), 4.30(\mathrm{dd}, \mathrm{J}=5.4 \mathrm{~Hz}, \mathrm{~J}=5.7 \mathrm{~Hz}$ 2H).

${ }^{13} \mathrm{C}$ NMR $\left(75 \mathrm{MHz}, \mathrm{CDCl}_{3}\right) \delta=41.1,47.2,51.2,52.4,53.2,56.4,63.2,86.8,154.7,166.3,167.5$, 168.3.

MS: $\mathrm{m} / \mathrm{z}(\%)=348\left(\mathrm{M}^{+}, 14.22\right), 350(5.16), 317(21.61), 319(7.34), 241(87.11), 176(100)$. 


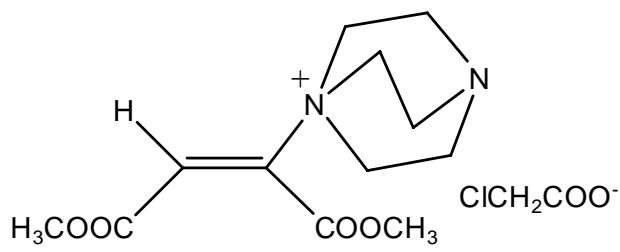



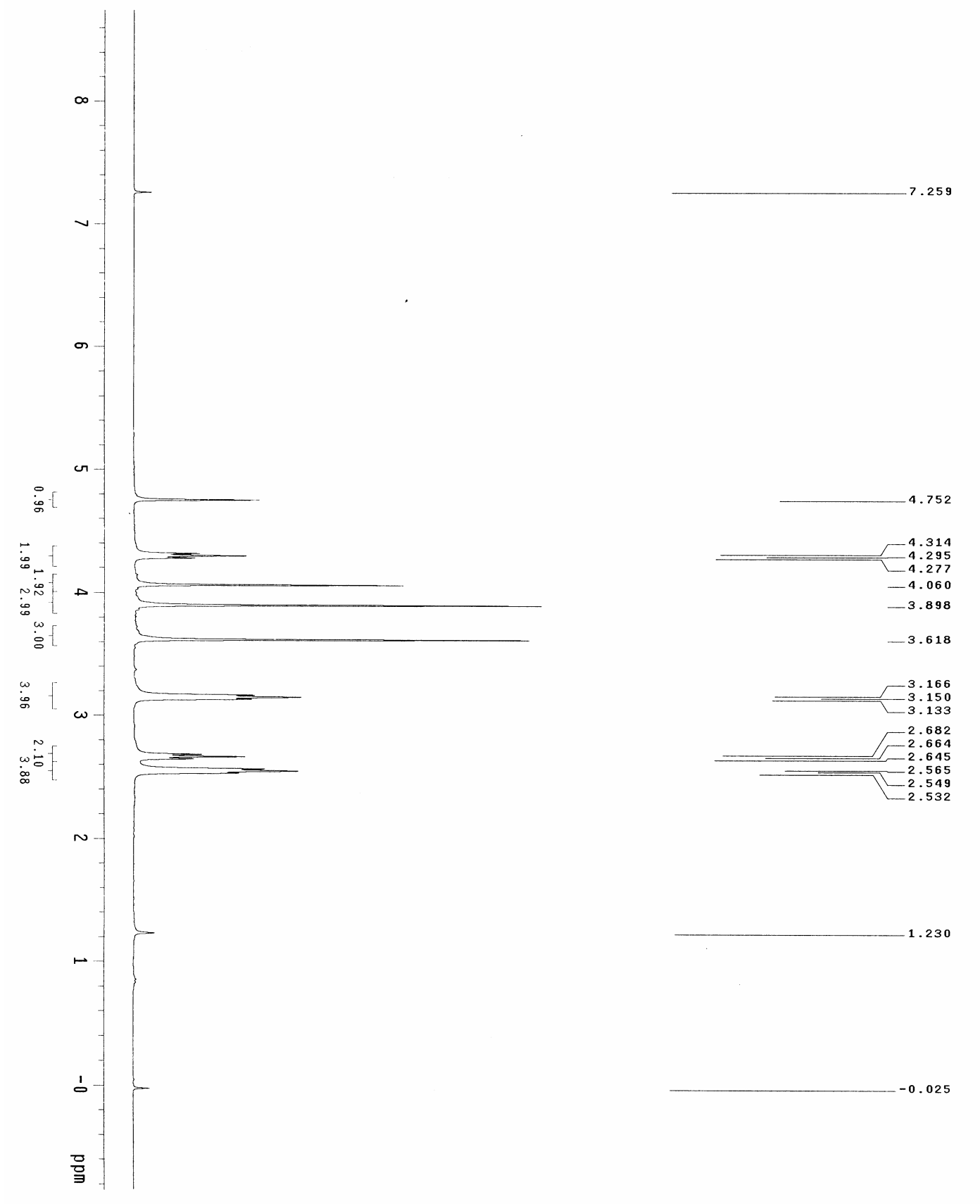


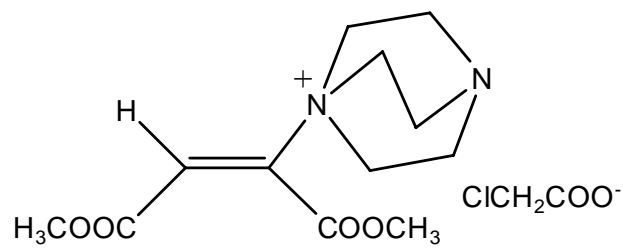



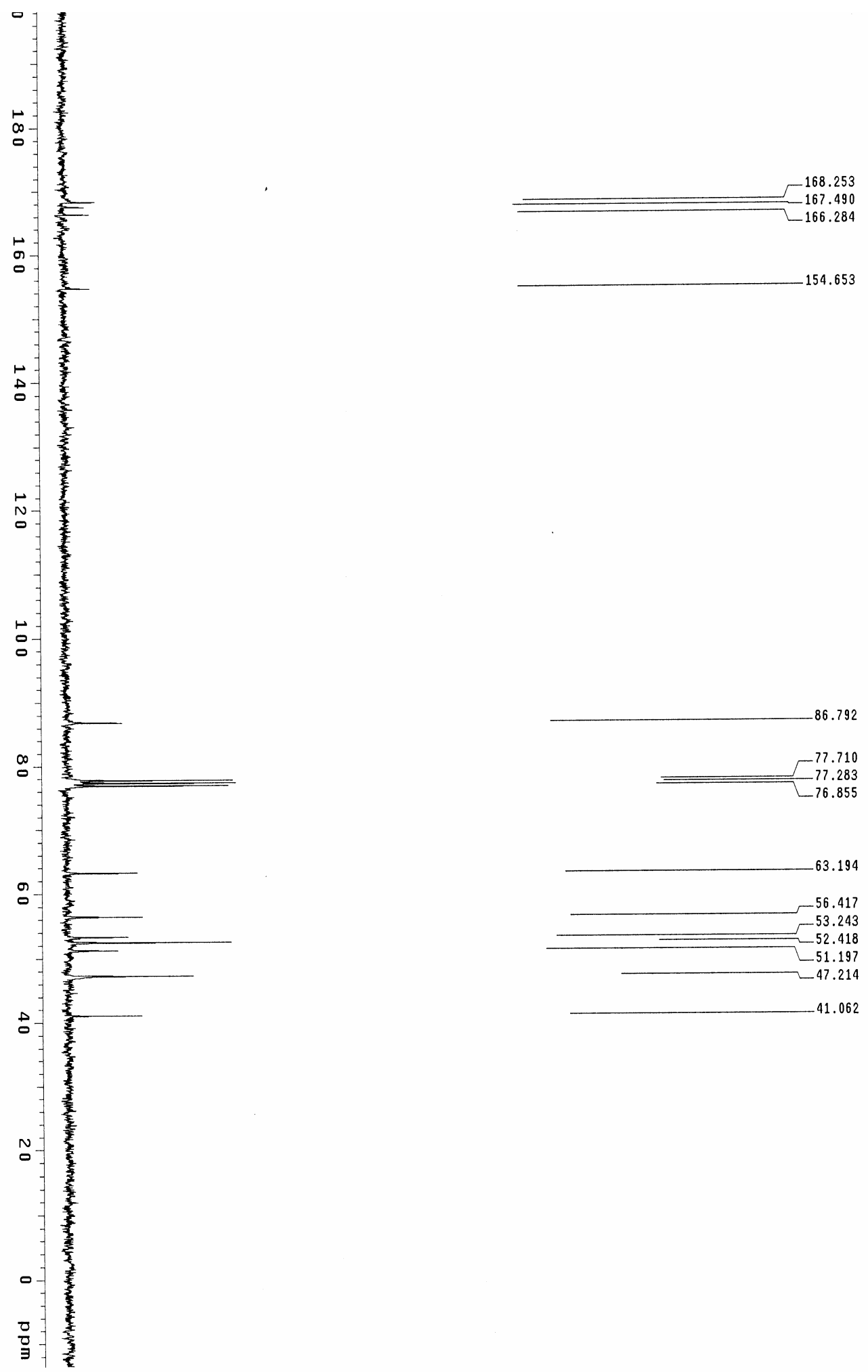

(III). Single-crystal X-ray crystallographic data for polySubstituted Furan 3c and 


\section{highly functionalized $2 H$-pyran 4 e.}

1. X-ray crystal structure of 3c

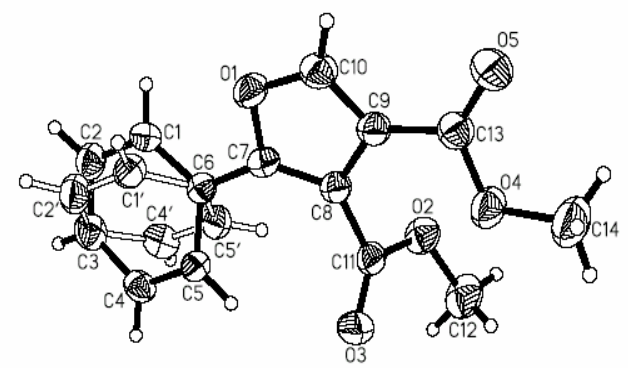

Crystal data for 3c: $\mathrm{C}_{14} \mathrm{H}_{12} \mathrm{O}_{5}, \mathrm{mp}: 68-70{ }^{\circ} \mathrm{C}$, chemical_formula_weigh: 260.24 , monoclinic space group P2 (1)/c, a=14.698(2), $b=11.720(1), c=7.3105(7) \AA ; \alpha=90.00^{\circ}, \beta=97.916(9)^{\circ}, \gamma=90.00^{\circ}, V=$ 1247.3(2) Å3. $T=290(2) \mathrm{K}, \mathrm{Z}=4, \mathrm{Dc}=1.386 \mathrm{Mg} / \mathrm{M}^{3}, \mu=0.106 \mathrm{~mm}^{-1}, \lambda=0.71073 \AA, \mathrm{F}(000) 544$, Crystal size $0.52 \times 0.48 \times 0.48 \mathrm{~mm}^{3}, 2255$ independent reflections [R (int) $=0.0122$ ], Reflections collected 2690: Refinement method, full-matrix least-squares on $\mathrm{F}^{2}$ : Goodness-of-fit on $\mathrm{F}^{2}$ 1.073:

Final $\mathrm{R}$ indices $[\mathrm{I}>2 \delta(\mathrm{I})] \mathrm{R}_{1}=0.0383, \mathrm{wR}_{2}=0.1038, \mathrm{R}$ indices (all data) $\mathrm{R}_{1}=0.0524, \mathrm{wR}_{2}=0.0138$. Extinction coefficient $0.126(7)$, Largest diff. peak and hole 0.173 and $-0.163 \mathrm{e} . \AA^{-3}$.

2. X-ray crystal structure of $4 \mathbf{e}$

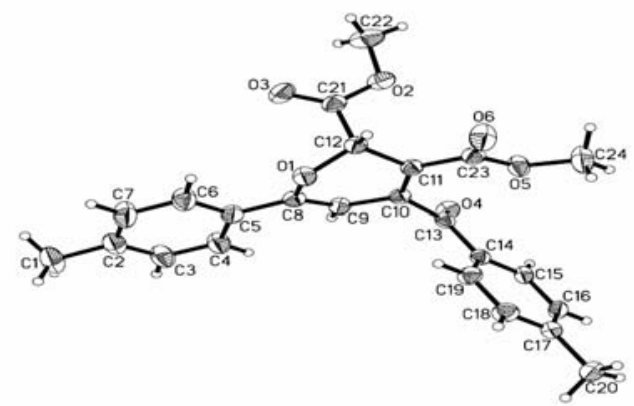

Crystal data for 4e: $\mathrm{C}_{24} \mathrm{H}_{22} \mathrm{O}_{6}, \mathrm{mp}: 148-150^{\circ} \mathrm{C}$, chemical_formula_weigh: 406.42 , monoclinic space group P2 (1)/c, $a=7.468(2), b=19.811(5), c=14.249(4) \AA ; \alpha=90.00^{\circ}, \beta=96.801(4)^{\circ}, \gamma=90.00^{\circ}, V=$ 
2093.2(10) А̊3. $T=291(2) \mathrm{K}, \mathrm{Z}=4, \mathrm{Dc}=1.290 \mathrm{Mg} / \mathrm{M}^{3}, \mu=0.093 \mathrm{~mm}^{-1}, \lambda=0.71073 \AA, \mathrm{F}(000) 856$, Crystal size $0.56 \times 0.45 \times 0.34 \mathrm{~mm}^{3}, 3872$ independent reflections [R (int) $=0.0168$ ], Reflections collected 10796: Refinement method, full-matrix least-squares on $\mathrm{F}^{2}$ : Goodness-of-fit on $\mathrm{F}^{2} 1.102$ :

Final $\mathrm{R}$ indices $[\mathrm{I}>2 \delta(\mathrm{I})] \mathrm{R}_{1}=0.0370, \mathrm{wR}_{2}=0.1028, \mathrm{R}$ indices (all data) $\mathrm{R}_{1}=0.0448, \mathrm{wR}_{2}=0.1064$.

Extinction coefficient 0.0088 (13), Largest diff. peak and hole 0.171 and -0.146 e. $\AA^{-3}$. 